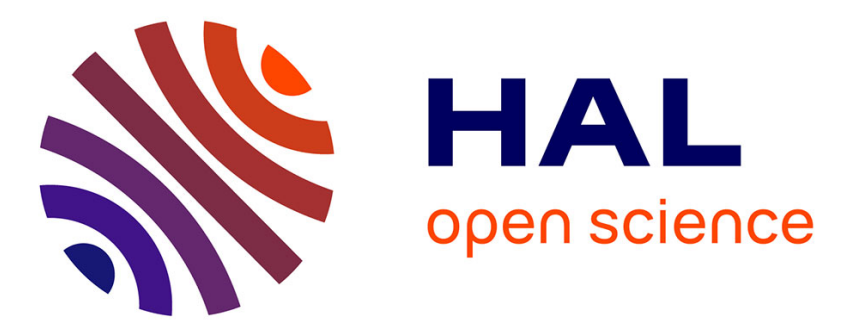

\title{
Stability, Convergence and Optimization of Interface Treatments in Weak and Strong Thermal Fluid-Structure Interaction
}

\author{
R. Moretti, Marc-Paul Errera, Vincent Couaillier, Frédéric Feyel
}

\section{- To cite this version:}

R. Moretti, Marc-Paul Errera, Vincent Couaillier, Frédéric Feyel. Stability, Convergence and Optimization of Interface Treatments in Weak and Strong Thermal Fluid-Structure Interaction. International Journal of Thermal Sciences, 2017, 126, pp.23 - 37. 10.1016/j.ijthermalsci.2017.12.014 . hal-01789013

\section{HAL Id: hal-01789013 \\ https://hal.science/hal-01789013}

Submitted on 9 May 2018

HAL is a multi-disciplinary open access archive for the deposit and dissemination of scientific research documents, whether they are published or not. The documents may come from teaching and research institutions in France or abroad, or from public or private research centers.
L'archive ouverte pluridisciplinaire HAL, est destinée au dépôt et à la diffusion de documents scientifiques de niveau recherche, publiés ou non, émanant des établissements d'enseignement et de recherche français ou étrangers, des laboratoires publics ou privés. 


\title{
Stability, Convergence and Optimization of Interface Treatments in Weak and Strong Thermal Fluid-Structure Interaction
}

\author{
Rocco Moretti $^{(1)}$, Marc-Paul Errera ${ }^{(*)}(2)$, Vincent Couaillier ${ }^{(3)}$, Frédéric Feyel ${ }^{(4)}$ \\ (1) ONERA, The French Aerospace Lab, France, Email: rocco.moretti@onera.fr \\ (2) ONERA, The French Aerospace Lab, France, Email: marc.errera@onera.fr \\ (3) ONERA, The French Aerospace Lab, France, Email: vincent.couaillier@onera.fr \\ ${ }^{(4)}$ SafranTech, Safran Group, France, Email: frederic.feyel@safrangroup.com
}

\begin{abstract}
This paper presents the stability, convergence and optimization characteristics of interface treatments for steady conjugate heat transfer problems. The Dirichlet-Robin and Neumann-Robin procedures are presented in detail and compared on the basis of the Godunov-Ryabenkii normal mode analysis theory applied to a canonical aero-thermal coupling prototype. Two fundamental parameters are introduced, a "numerical" Biot number that controls the stability process and an optimal coupling coefficient that ensures unconditional stability. This coefficient is derived from a transition of the amplification factor. A comparative study of these two treatments is made in order to implement numerical schemes based on adaptive and local coupling coefficients, with no arbitrary relaxation parameters, and with no assumptions on the temporal advancement of the fluid domain. The coupled numerical test case illustrates that the optimal Dirichlet-Robin interface conditions provide effective and oscillation-free solutions for low and moderate fluidstructure interactions. Moreover, the computation time is slightly shorter than the time required for a CFD computation only. However, for higher fluid-structure interactions, a Neumann interface condition on the fluid side presents good numerical properties so that no relaxation coefficients are required.
\end{abstract}

Keywords - Conjugate heat transfer, optimal coefficients, Dirichlet, Neumann, Robin, stability

\section{Nomenclature}

$a \quad$ thermal diffusivity $\left[\mathrm{m}^{2} \cdot \mathrm{s}^{-1}\right]$

$B i \quad$ Biot number

$B i^{(\Delta)}$ mesh Biot number

$B i_{v} \quad$ numerical Biot number

$D \quad$ Fourier number

$\bar{D} \quad$ normalized Fourier number

$F \quad$ inviscid and viscous flux

$g \quad$ temporal amplification factor

$h$ heat transfer coefficient $\left[\mathrm{W} \cdot \mathrm{m}^{-2} \cdot \mathrm{K}^{-1}\right.$ ]

$n \quad$ coupling iteration

$\varepsilon \quad$ error tolerance

$\lambda$ thermal conductivity $\left[\mathrm{W} \cdot \mathrm{m}^{-1} \cdot \mathrm{K}^{-1}\right]$

$K \quad$ thermal conductance $\left[\mathrm{W} \cdot \mathrm{m}^{-2} \cdot \mathrm{K}^{-1}\right]$

$N \quad$ number of cells at interface cells

$q$ heat flux [W.m ${ }^{-2}$ ]

$t \quad$ time [s]

$T$ temperature [K]

$\Gamma \quad$ thermal conductivity matrix

w fluid conservative quantity

$y+\quad$ non-dimensional wall distance

$z \quad$ complex variable

$\alpha \quad$ coupling coefficient $\left[\mathrm{W} \cdot \mathrm{m}^{-2} \cdot \mathrm{K}^{-1}\right]$

$\kappa \quad$ spatial amplification factor

$v \quad$ FVM/FEM parameter

$\begin{array}{ll}\rho & \text { density }\left[\mathrm{Kg} \cdot \mathrm{m}^{-3}\right] \\ \Lambda & \text { characteristic size [m] } \\ \Omega & \text { domain/partition } \\ \Delta y & \text { size 1st cell [m] } \\ \Delta t & \text { time step [s] }\end{array}$

$\underline{\text { Subscripts }}$

$c \quad$ coupled

$f \quad$ fluid domain

$s \quad$ solid domain

ref reference value

$v \quad$ numerical

$v_{f} \quad$ inward unit normal to the fluid domain

$v_{s} \quad$ inward unit normal to the solid domain

\begin{tabular}{ll}
\multicolumn{2}{l}{ Superscripts } \\
$n n$ & temporal index \\
min & minimum \\
max & maximum \\
opt & optimal
\end{tabular}

(ڤ) unknown value 


\section{Introduction}

Conjugate heat transfer (CHT) analysis is a simulation process that addresses the thermal interaction between a body and a fluid flowing over or through it. Conjugate heat transfer problems occur whenever fluid convection and solid material conduction are taken into account simultaneously. The concept of "conjugated problems" was first formulated in the early 1960s by Perelman [1]. As a result, heat transfer has been often investigated as a coupled problem [2] since this mutual interaction has become increasingly important in many numerical simulations.

CHT analysis can be performed in a monolithic manner in which the equations are solved simultaneously in a single solver [3][4] but such an approach is not flexible and cannot be pursued with commercial codes. In contrast, partitioned techniques allow the direct use of a specialized solver for each subdomain, offering significant benefits in terms of efficiency and code reuse. In this strategy, the solution is advanced in time separately within each partition [5][6][7].

However, the time lag due to the sequential treatment in partitioned procedures can have a detrimental effect on the stability and performance leading to slow convergence. In a fluid-structure interaction (FSI), this staggered process generally leads to spurious energy production. Specific numerical treatments are proposed in the literature to overcome these difficulties. Examples include a combined interface boundary condition, proposed by Jaiman et al. [8][9], an interface correction controlled by a coupling parameter [10] and the use of a specific partitioned algorithm in conjunction with a relevant Robin condition [11].

In CHT, we experience the same problems and constraints. There are many similarities between FSI and CHT. A variety of approaches have been employed based on finite elements, finite volumes, boundary elements and spectral approximations [12]-[17]. Numerical methods are also required to counteract the intrinsic destabilizing effect of the time lag and time discrepancy between each sub-domain. It is likewise standard to enforce continuity at the interface between the fluid and the solid. This can be achieved by using one or two coupling coefficients that control stability. Many papers in CHT have sought to improve the interface conditions by adopting simple model problems from which the interface conditions and coupling coefficients can be derived.

In many cases, the model problems show that the structure of complex multiphysics systems is often as important as the behavior of the individual components themselves. Indeed, fluid and solid domains can interact in many different ways. Model problems are a means to understand and quantify these dynamic interactions. For instance, in FSI, the added-mass was highlighted by Causin et al. [18] from a simplified model problem. Using a different model problem and a normal mode analysis, Banks and Sjögreen [19] obtained a similar result. Similarly, in CHT, the nature of the instabilities derived from a simplified 1D model provides insights into the potential instabilities in $2 \mathrm{D} / 3 \mathrm{D}$ flows.

The behavior of the interface conditions in CHT is also often studied using a normal mode analysis. For instance, the pioneering work of Giles [20], the new procedure applied to CHT proposed by Roe et al. [21], the composite grid solver introduced by Henshaw and Chand [22] and the stability analysis in transient CHT presented by Kazemi-Kamyab et al. [23]. An interesting alternative is the steady-state approach described by Verstraete and Scholl [24]. It should be mentioned that there are other methods of investigation, such as the energy method, to analyze wellposedness and stability [25]. 
By using a thermal model problem, Errera and Chemin [26] have identified a numerical transition that can be expressed mathematically. This fundamental result has been derived from a normal mode stability analysis based on the theory of Godunov-Ryabenkii [27][28][29]. This transition results in an optimal coefficient in terms of stability and convergence.

The formulation of the optimal coefficient was published relatively recently. Consequently few CHT computations have been reported, that take advantage of it. However, some interesting results have been reported. For instance, in a steady CHT computation of an effusion cooling system [30], the CPU time necessary to converge was divided by a factor of ten, in contrast to a conventional method. In another work devoted to testing systematically the values of various coupling coefficients [31], it was shown that the optimal coefficient in combination with a Dirichlet-Robin procedure (temperature prescribed to the fluid sub-domain) could be applied in an efficient manner as a tool for predicting and obtaining excellent stability properties. This result was confirmed recently in a complex set-up of a heating cell found in various industrial applications (conveyors, reheat furnaces). A systematic comparison of various coefficients was undertaken and it was shown that the optimal coefficient outperformed the previous results in the literature [32]. In transient CHT problems, optimal coefficients can also be applied to analyze heat transfer during a full transient flight cycle as shown in [33] where specific numerical characteristics at the interface were provided.

Previous studies suggest that the one-dimensional normal mode analysis could provide relevant coefficients directly applicable to industrial CHT problems. These promising results have been obtained by using Dirichlet-Robin conditions, a method widely used in the literature. However, ideally, Robin conditions on either side of the interface should be considered because they introduce local simplified models whether for FSI [18] [34] or CHT [35]. Yet, this general Robin-Robin interface condition results in a very large family of schemes and we prefer, as a first step, to consider the two conditions that form the basis of this general approach. Thus, the present paper is confined to two complementary interface treatments :

- A Dirichlet-Robin procedure : the temperature obtained from the solid is applied on the fluid side, and a "relaxed heat flux" is in turn used as a boundary condition for the solid.

- A Neumann-Robin procedure : the heat flux obtained from the solid is applied on the fluid side and a Robin condition is in turn used as a boundary condition for the solid.

The above CHT interface procedures are the most commonly used conditions in the literature. The goal of this paper is to present them in detail and to provide, for the first time, their remarkable properties, in particular the temporal and spatial amplification factor, instability zones, upper and lower stability bounds and optimal coefficients on the basis of a canonical coupling prototype. These results will be summarized in tables where the numerical properties are evaluated according to the nature of the fluid-solid interaction. Moreover, a comparative study of these two treatments will be made in order to implement efficient numerical schemes, that is to say schemes based on adaptive and local coupling coefficients, with no arbitrary relaxation parameters and with no assumptions on the temporal progression of the fluid domain.

The paper is composed as follows. The theoretical study is presented first (Section 2) and the precise conditions to obtain optimal coefficients are provided. Then, the numerical Dirichlet-Robin transmission procedure is described: The advantages and disadvantages of this specific scheme are presented along with the appropriate numerical treatments for optimization (Section 3). To enhance further the numerical efficiency of the coupled approach, we put forward another complementary 
numerical alternative, a Neumann-Robin procedure and the remarkable properties of this condition are provided for the first time (Section 4). These two interface procedures are then briefly compared (Section 5). This strategy is illustrated via a CHT test case (Section 6), emphasizing the stability and convergence properties of the coupling schemes under weak/moderate (Section 7) and strong (Section 8) thermal fluid-structure interaction.

\section{GOVERNING EQUATION AND COUPLING MODEL}

\subsection{Finite volume fluid solver}

In the fluid domain, $\Omega_{f}$, the Reynolds-Averaged Navier-Stokes (RANS) equations are solved. The governing equations are the time-dependent Navier-Stokes (NS) equations which express the conservation laws written in the conservation form as

$$
\frac{\partial \mathbf{w}_{f}}{\partial t}+\nabla \cdot\left[F\left(\mathbf{w}_{f}\right)\right]=0 \quad \text { in } \Omega_{f}
$$

where $\mathbf{w}_{f}$ represents the vector of mass, momentum and energy quantities, $F$ represents the flux including inviscid and viscous terms. The inviscid terms are solved using a second-order upwind spatial discretization. The viscous terms are discretized with a five-point central difference formulation. The time integration is obtained by an implicit method.

\subsection{Finite element solid solver}

In the solid domain, $\Omega_{s}$, if there are no heat sources, the steady temperature is modeled as a balance of thermal transport governed by the conductive heat transport equation

$$
\nabla \cdot(\Gamma \cdot \nabla T)=0 \quad \text { in } \Omega_{s}
$$

where $T=T(x, y, z, t)$ is the unknown temperature field and $\Gamma$ is the thermal conductivity matrix. Assuming the thermal conductivity to be constant, the steady diffusion equation in the solid is reduced to the Laplace equation $\Delta T=0$, i.e. an elliptic PDE over $\Omega_{s}$.

\subsection{Partitioned CHT procedure}

The partitioned CHT strategy employed hereafter is motivated by the desire to obtain a fast and stable steady solution to the fluid-structure thermal problem. The basic conventional serial staggered (CSS) algorithm, composed of 4 steps (see Figure 1) was implemented here to sequentially execute the CFD and the conduction solver. However, other partitioned fluid-thermal schemes could also be used [38]. 
$\Omega_{f}$

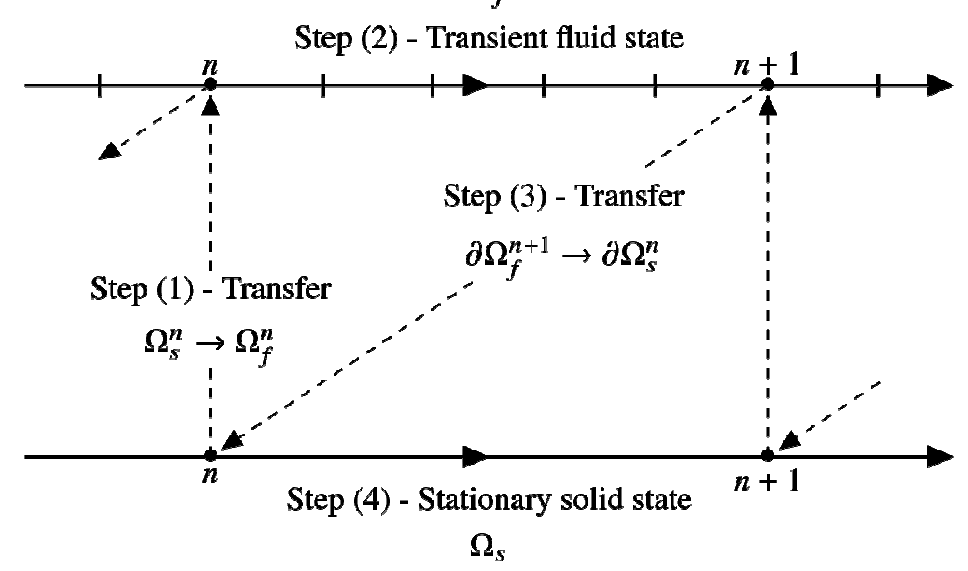

Figure 1. CSS (Conventional Serial Staggered) algorithm

\subsection{Temporal steady coupling strategy}

The fluid and solid domains operate on different time scales and the thermal response of the solid is generally very long. As a result, if only a fluid-solid steady solution is sought, the numerical coupling strategy should not take into account the unsteady diffusion of the solid material that causes no effect on the steady solution. So, in the solid, the steady solution of the heat equation can be obtained easily and directly from the Laplace equation. On the contrary, in a fluid domain, the steady-state NavierStokes equations (laminar or RANS) are generally solved by a time-marching scheme. As a result, if the two above-mentioned approaches perform well as single sub-systems they should be taken together in a coupled system. This means that the intermediate temporal solutions are not physically meaningful. This "steady strategy" is adopted in the current paper. Note that this is a commonly used fluid-solid methodology when only a steady state is sought [24][26][36][37]. This approach does require however a special attention to stability issues.

\subsection{Coupling model}

The coupling model is composed of two partitions with a shared interface. A schematic of both domains with node numbering is shown in Figure 2 (solid $y \leq 0$, fluid $y \geq 0$ ). A uniform grid on either side of the interface is employed. The boundary conditions at this interface are presented in the next paragraph.

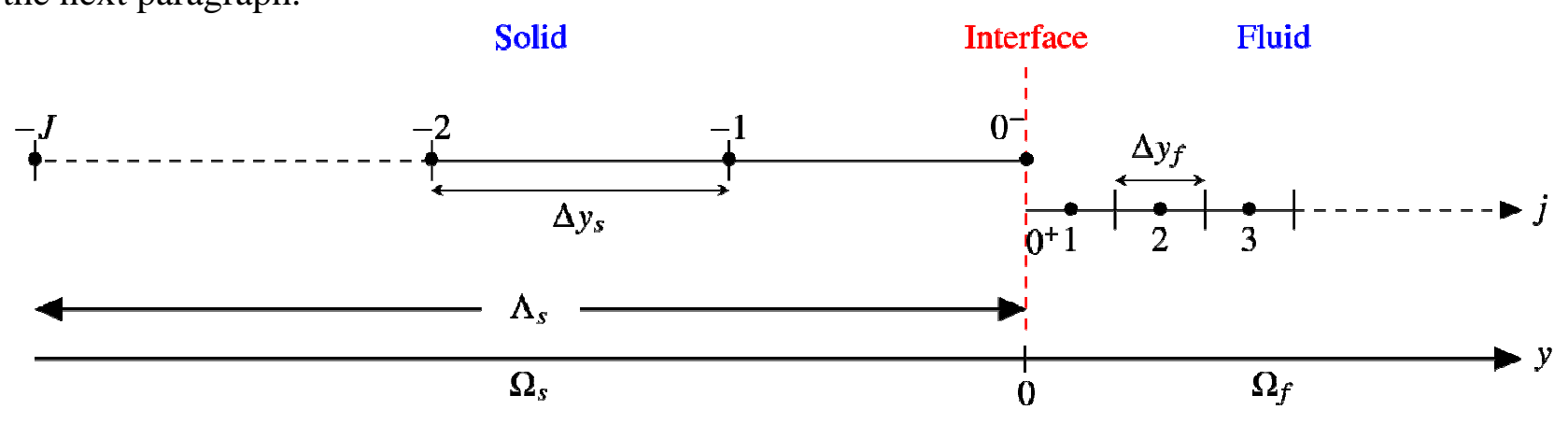

Finite Element Method

Finite Volume Method

Figure 2. Problem discretization for the 1D model (solid $y \leq 0$, fluid $y \geq 0$ ). 


\subsection{Interface conditions}

Assuming that the convection term is negligible in the first fluid cell (no velocity component normal to the F-S interface in 2D and 3D flows), the CHT problem may be modelled through a simple one-dimensional thermally coupled problem. The equations in both domains are then solved through the CSS algorithm, with interface data being exchanged at each coupling iteration.

At the F-S interface a general Robin transmission condition on the solid side, leads to the equation

$$
\hat{q}_{s}+\alpha_{f} \hat{T}_{s}=-q_{f}+\alpha_{f} T_{f}
$$

where $q$ is the normal heat flux, $T$ is the temperature and $\alpha$ is a coupling coefficient; the superimposed hat symbol $\left(^{\wedge}\right)$ denotes the sought values. Note that $q_{s}=-K_{s} . \partial T_{s} / \partial v_{s}$ is the normal solid heat flux and $q_{f}=-K_{f} . \partial T_{f} / \partial v_{f}\left(v_{f}\right.$ and $v_{s}$ are inward-pointing unit normals, $\left.v_{f}=-v_{s}\right)$ where $K$ is the thermal conductance.

Analogously, the same type of interface condition can be defined on the fluid side :

$$
\hat{q}_{f}+\alpha_{s} \hat{T}_{f}=-q_{s}+\alpha_{s} T_{s}
$$

Applying the right hand side of Eq.(4) as a boundary condition on the fluid domain, might pose a technical challenge for most research and commercial CFD packages that employ a reference temperature. As a result, Eq.(4) can easily be rewritten in the following form

$$
\hat{q}_{f}=\alpha_{s}\left(\left[-\frac{q_{s}}{\alpha_{s}}+T_{s}\right]-\hat{T}_{f}\right)
$$

\subsection{Temporal and spatial amplification factors}

The Godunov-Ryabenkii theory is used to analyze the stability of the coupled fluid-solid procedure because it includes the effects of boundary conditions on the numerical stability of the problem.

The first step of the stability analysis consists in introducing normal mode solutions in the discrete model equations. These are the transient energy equation in the fluid and the steady heat conduction in the solid, with Robin conditions on both sides of the interface. The second step consists in considering the eigensolutions of the discrete problem. After elementary transformations not reported in this paper (see details in [26]), the temporal amplification factor $g(z)$ (i.e. each mode increases in amplitude by the ratio $|g(z)|$ ) can be expressed as follows

$$
z=g(z)=\frac{\left(\alpha_{f}+\alpha_{s}\right) K_{f}}{\left(K_{f}+\alpha_{s}\right)\left(K_{s}+\alpha_{f}\right)} \kappa_{f}+\frac{\left(K_{f}-\alpha_{f}\right)\left(K_{s}-\alpha_{s}\right)}{\left(K_{f}+\alpha_{s}\right)\left(K_{s}+\alpha_{f}\right)}
$$

where $K_{f}=\lambda_{f} / v \Delta y_{f}(v=1 / 2$ in a FVM and $v=1$ in a FEM $)$ and $K_{s}=\lambda_{s} / \Lambda_{s}$ are the thermal fluid and solid thermal conductances, respectively. The temporal amplification factor depends upon the complex function $\kappa_{f}=\kappa_{f}\left(z, D_{f}\right)$ : 


$$
\kappa_{f}=\kappa_{f}\left(D_{f}, z\right)=1+\frac{z-1}{2 D_{f} z}-\sqrt{\left(1+\frac{z-1}{2 D_{f} z}\right)^{2}-1}
$$

This function is thus solution of a quadratic equation obtained from the interior scheme $(j>0)$ in the fluid domain [26]. $D_{f}$ denotes the mesh Fourier number defined by

$$
D_{f}=a_{f} \Delta t_{c} / \Delta y_{f}^{2}
$$

where $a_{f}$ is the fluid thermal diffusivity and $\Delta t_{c}$ is the coupling time step. The mesh Fourier number characterizes the heat conduction in the layer mesh of the transient domain.

For simplicity, we define the function $\bar{D}_{f}$ representing a normalized Fourier number in terms of $D_{f}$

$$
\bar{D}_{f}=\kappa_{f}(z=-1)=\frac{D_{f}}{1+D_{f}+\sqrt{1+2 D_{f}}}
$$

$D_{f}$ describes the interval $\left[0,+\infty\left[\right.\right.$ as $\bar{D}_{f}$ ranges over $[0,1[$.

\subsection{Stability analysis and transition}

As can be seen from (6) and (7), the complex function $g$ is a complicated nonlinear equation in $z$. According to the normal mode stability theory, the approximation is stable only if no nontrivial solutions to the equation $z=g(z)$ exist for $|z| \geq 1$. Using the corollary of the maximum modulus principle in complex analysis, it can be shown that the condition $z \neq g(z)$ for $|z| \geq 1$ is automatically satisfied if $\max _{|z|=1}\{|g|\}<1$. In addition, the maximum $\max _{|z|=1}\{|g|\}$ is obtained either at the points $z=+1$ or $z=-1$ (see [26][31]). Moreover, when "specified conditions" are met, there is a sudden transition from one point to another resulting in an amplification factor composed of two half-lines with a singular point at the intersection of these two lines. At this intersection the amplification factor turns back and attains an absolute minimum that is always located in the stable zone. The point where the maximum is transferred is a fundamental transition in aerothermal coupling. 


\section{DIRICHLET-ROBIN INTERFACE CONDITIONS}

\subsection{Stability behavior}

The Dirichlet-Robin conditions $\left\{\alpha_{f} \geq 0 ; \alpha_{s}=\infty\right\}$ in Eqs. (3) and (4) are considered in this section. These conditions are frequently used in the literature. This procedure is also referred to as the coefficient forward temperature back ( $h$ FTB) method [24] [37][39].

\subsection{Optimal coefficient}

The existence of a transition value for $\alpha_{f}$ is highlighted. At this transition, the shape of the curve changes, resulting in the lowest amplification factor. This value, denoted $\alpha_{f}^{(o p t)}$, is given by

$$
\alpha_{f}^{(o p t)}=\frac{K_{f}}{2}\left(1-\bar{D}_{f}\right)
$$

Note that this coefficient exists unconditionally. In other words, there is always, in the DirichletRobin procedure, a positive coefficient $\alpha_{f}$ satisfying Eq.(10).

\subsection{Mesh Biot number}

The stability condition $\left|g\left(z, \alpha_{f}, \alpha_{s}=\infty\right)\right|<1$ applied to Eq. (6) leads to the following lower stability bound

$$
\alpha_{f}^{\min }=\frac{K_{f}}{2}\left(1-\bar{D}_{f}\right)-\frac{K_{s}}{2}
$$

which can also take the form

$$
\alpha_{f}^{\min }=\frac{K_{s}}{2}\left[B i^{(\Delta)}\left(1-\bar{D}_{f}\right)-1\right]
$$

In this equation, we have introduced the dimensionless number

$$
B i^{(\Delta)}=\frac{K_{f}}{K_{s}}=\frac{\lambda_{f} /\left(v \Delta y_{f}\right)}{\lambda_{s} / \Lambda_{s}}=\frac{\text { thermal conductance of the 1st fluid cell }}{\text { thermal conductance of the solid domain }}
$$

$B i^{(\Delta)}$ may be regarded as a mesh Biot number (or local Biot number) representing the thermal fluidstructure interaction.

\subsection{Numerical Biot number}

We can now introduce another dimensionless number $B i_{v}$ :

$$
B i_{v}=B i^{(\Delta)}\left(1-\bar{D}_{f}\right)
$$

This parameter takes into account the coupling time step via the Fourier number. This dimensionless number is defined at any coupling iteration of a CHT computation and results from a balance between the unsteady fluid and steady solid domain properties. $B i_{v}$ is a local representation of the thermal fluid-structure coupling and plays a key role in the stability analysis. In the literature, 
a conventional Biot number is used, requiring a heat transfer coefficient to be defined at steady state. We will explain the difference between these two numbers in subsection 3.6 .

From Eq. (12) and (14), two zones can be considered. The first zone is defined by

$$
B i_{v}=B i^{(\Delta)}\left(1-\bar{D}_{f}\right) \leq 1
$$

When this condition holds, the CHT procedure is stable for any positive value of $\alpha_{f}$, in particular for $\alpha_{f}=0$. Thus, a Dirichlet-Neumann transmission condition can be used with no relaxation and without affecting the stability of the coupled problem.

The second zone is

$$
B i_{v}=B i^{(\Delta)}\left(1-\bar{D}_{f}\right) \geq 1
$$

When this condition holds, the CHT procedure is inherently prone to instability and a relaxation coupling coefficient must be used such that $\alpha_{f}>\alpha_{f}^{\min }$.

The function $\max \{|g|\}$, i.e. the maximum value the amplification factor may take, is plotted in Figure 3 as a function of the coupling coefficient $\left(\alpha_{f} \geq 0\right)$, for two different mesh Biot numbers and the same Fourier number $\bar{D}_{f}\left(\bar{D}_{f}=0.53\right)$. Note that this function is defined and continuous and that each curve is composed of two half-lines with a singular point similar to a cusp at the intersection. At $\alpha_{f}=\alpha_{f}^{(o p t)}$, the two branches of the amplification factor join. At this remarkable value, the amplification factor attains its absolute minimum. 


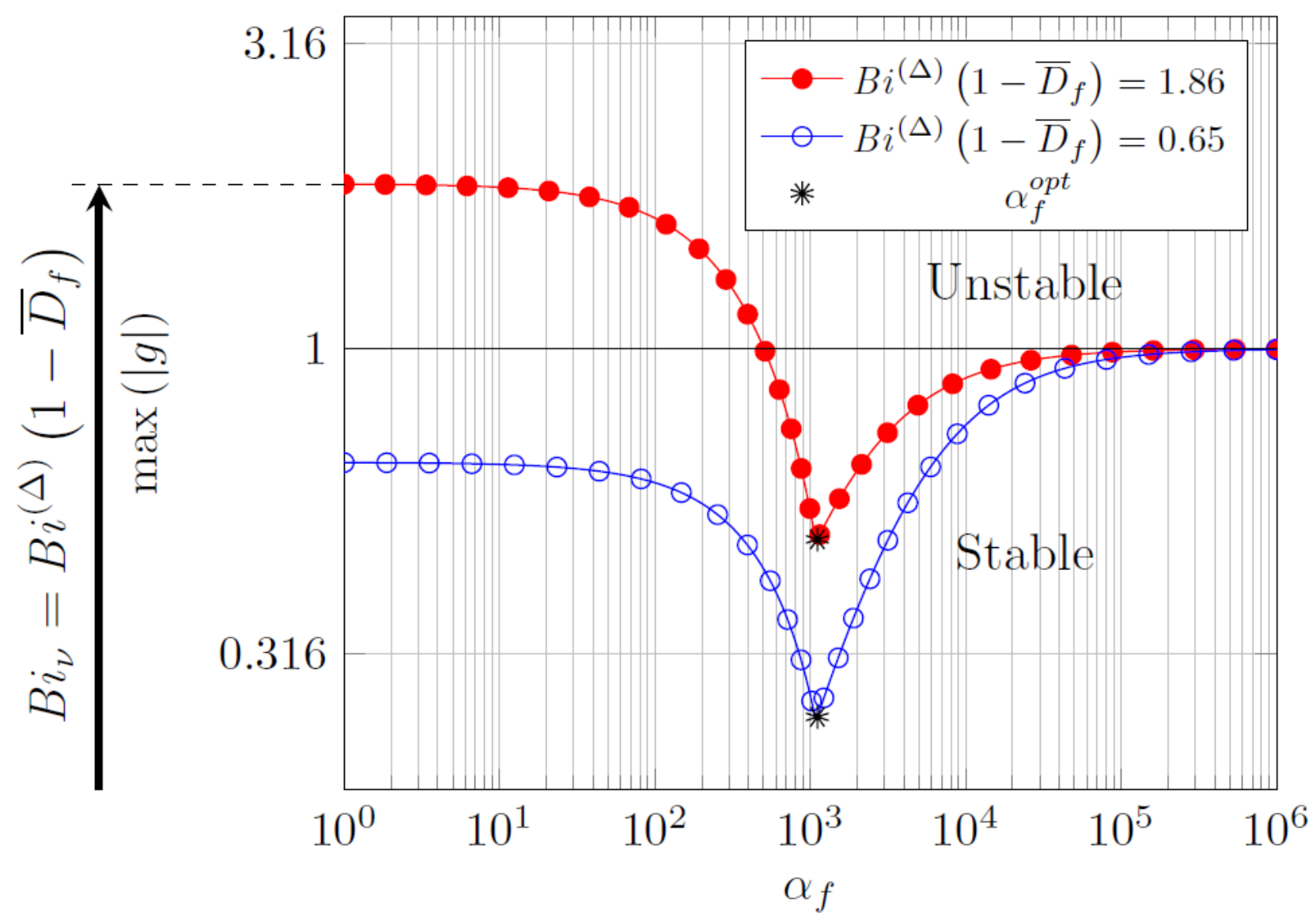

Figure 3. Temporal amplification factor of Dirichlet-Robin condition for mesh Biot numbers Blue curve : unconditionally stable (low Fluid-Structure interaction) and optimal coefficient Red curve : conditionally stable (higher F-S interaction) and optimal coefficient

Figure 3 illustrates the general trends just discussed, for two different numerical Biot numbers.

The main results of this stability analysis are summarized in Table 1. At low or moderate values of $\alpha_{f}$, the amplification factor is partly outside the stability domain for $B i_{v}=1.86$ (first row in Table 1) and completely inside the stability domain for $B i_{v}=0.65$ (second row in Table 1). The fundamental role of $\alpha_{f}^{(o p t)}$ in controlling and guiding the behavior of the two curves is highlighted. 


\begin{tabular}{|l|l|l|l|l|l|l|l|l|}
\hline$\alpha_{f}$ & 0 & & $\alpha_{f}^{\text {min }}$ & & $\alpha_{f}^{o p t}$ & & $\infty$ & $\mathbf{c o ~ n ~ d ~ i ~ t ~ i ~ o ~ n ~}$ \\
\hline$|g|$ & $g^{0}$ & U N S T A B L E & 1 & & $g^{o p t}$ & & 1 & $B i^{(\Delta)}\left(1-\bar{D}_{f}\right) \geq 1$ \\
\hline$|g|$ & $g^{0}$ & $\longrightarrow$ & $g^{o p t}$ & & 1 & $B i^{(\Delta)}\left(1-\bar{D}_{f}\right) \leq 1$ \\
\hline & $g^{0}=B i^{(\Delta)}\left(1-\bar{D}_{f}\right)$ & $g^{o p t}=\frac{B i^{(\Delta)}}{2}\left(1-\bar{D}_{f}\right) / 1+\frac{B i^{(\Delta)}}{2}\left(1-\bar{D}_{f}\right)$ \\
\hline
\end{tabular}

Table 1 : Numerical behavior of Dirichlet-Robin procedure vs $\alpha_{f}\left(\alpha_{s}=\infty ; \alpha_{f} \geq 0\right)$

The mathematical expression of the optimal coefficient was already presented in [26][31]. However, the other numerical properties of the Dirichlet-Robin transmission conditions (Table 1) have never been published before.

Table 1 shows that, as long as the transient effects are predominant, the unconditional stability of the coupling procedure without relaxation can always be ensured by increasing $D_{f}$ (and hence $\bar{D}_{f}$ ) such that condition (15) holds. Otherwise, a certain level of relaxation is needed and the optimal level is provided by the choice of the optimal coefficient (Eq. (10)).

\subsection{Numerical Biot number and nature of the interface treatment}

The theoretical results proposed in this paper have the widest practical effect of reducing the CPU time and ensuring unconditional stability. Indeed, the following amplification factor is obtained

$$
\left|g\left(z, \alpha_{f}^{(o p t)}\right)\right|=\frac{\alpha_{f}^{(o p t)}}{K_{s}+\alpha_{f}^{(o p t)}}<1
$$

As a result, the optimal coefficient always provides a stable procedure and the lowest amplification factor.

\subsection{Conventional and numerical Biot number}

The current study introduces a "numerical" Biot number and particular emphasis was placed on its key role in the stability process. This numerical Biot number takes into account the thermal response of the first point of the boundary layer and directly participates in the stability of the coupled process (see Figure 4). It is worth noting that diffusion, on the fluid side, dominates and guides the coupling process, as long as transient effects prevail.

The conventional Biot number defined by

$$
B i=\frac{h}{K_{s}}
$$


is a criterion that gives a direct indication of the relative importance of the conduction and convection processes. It measures the resistance to heat flow within the solid relative to the resistance presented by the convection processes at the surface (see Figure 4). As a result, this number is a key parameter that determines the stability of the F-S equilibrium [24] [31]. However, this parameter, which strictly speaking is not defined during the fluid transients, cannot be used to set up a numerical CHT procedure, as long as a transient fluid state is involved in this procedure.

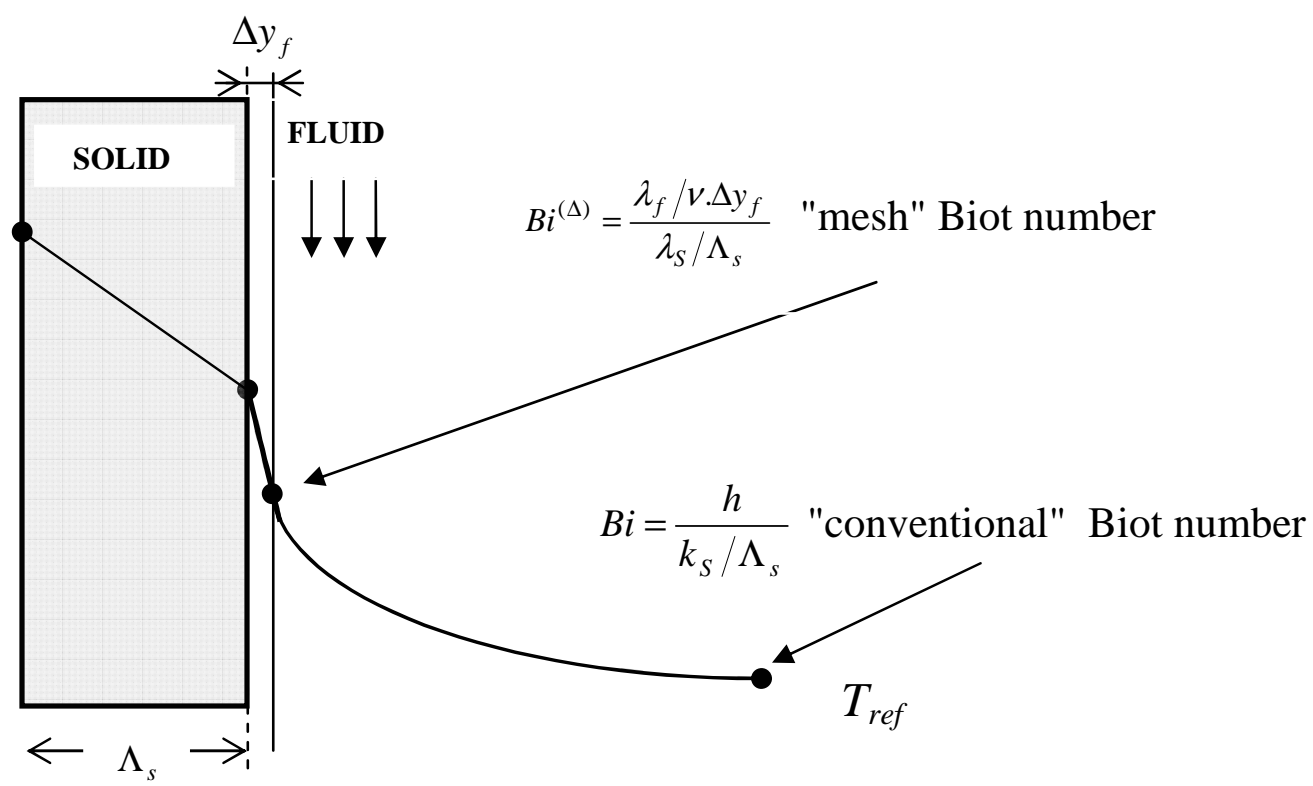

Figure 4 - Mesh and conventional Biot numbers

\subsection{The "optimal" coefficient : a dynamic approach}

As already mentioned, the Dirichlet-Robin procedure is widely used but the success of its application depends on finding a relevant coupling coefficient. It is often pointed out that a trade-off between computing time and stability must be considered. The results reported in this paper allow us to analyze the advantages and disadvantages of the effects of any other coupling coefficient of the Dirichlet-Robin condition. In the literature, various methods or values for this coefficient are presented.

As long as fluid transient effects are predominant, it may be tempting to employ the fluid temperature in the first grid cell as a reference temperature. As a result, the local coupling coefficient becomes

$$
\alpha_{f}=K_{f}
$$

This coefficient, proposed by Heselhaus [40], is always located in the stability zone (right side of the curves in Figure 3). However, it is a static coefficient that does not take into account the transient effect - represented by $\left(1-\bar{D}_{f}\right)$ - of the fluid flow between two coupling instants. Thus, this coefficient over-emphasizes the stability component of the coupling process compared with its computational time, especially for a large Fourier number (large coupling time step), and results most often in a prohibitive CPU time. 
Without the assistance of a mathematical tool, such as a stability analysis, the choice of a relaxation parameter is made through trial and error. A small value of the coefficient will provide a large change in the wall temperature at each coupling and can lead to a faster procedure. However, Eq (12) shows that a coupling coefficient must be larger than $\alpha_{f}^{\min }$, in other words, higher than the difference between the "transient" thermal conductance $K_{f}\left(1-\bar{D}_{f}\right)$ of the flow and the static solid thermal conductance $K_{s}$.

Two points should be noted :

(1) In CHT, the ratio of the thermal conductivities $\lambda_{f} / \lambda_{s}$ plays an important role. However, this ratio does not take the characteristic lengths of the two media into account. It is therefore preferable to introduce the mesh Biot number defined by (13).

(2) In addition to the above considerations, the stabilizing effect of the coupling time step is the result of the Fourier number (Eq.(8)) or its normalized version (Eq. (9)). The combination of this term and the mesh Biot number provides the numerical Biot number (Eq. (14)) that can also be interpreted as the $y$-intercept of the amplification factor curves shown in Figure 3. It can also be regarded as a dynamic measure of the strength of the "transient" thermal F-S interaction. This number is a key factor in the stability analysis. 


\section{NEUMANN-ROBIN INTERFACE CONDITIONS}

\subsection{Stability bounds}

The Neumann-Robin conditions $\left\{\alpha_{f} \geq 0 ; \alpha_{s}=0\right\}$ in Eqs (3) and (4) are considered in the following. This procedure is also referred to as the coefficient forward flux back $(h \mathrm{FFB})$ method [24][37][39]. The stability condition $\left|g\left(z, \alpha_{f}\right)\right|<1$ applied to Eq. (6) leads, after some basic calculus manipulations, to an upper stability bound $\alpha_{f}^{\max }$

$$
\alpha_{f}^{\max }=\frac{2 K_{s} K_{f}}{K_{s}-K_{f}\left(1+\bar{D}_{f}\right)}=\frac{2 K_{f}}{1-B i^{(\Delta)}\left(1+\bar{D}_{f}\right)}
$$

The coupling coefficient is always positive. As a result, two stability regions are highlighted :

- $B i^{(\Delta)}\left(1+\bar{D}_{f}\right) \geq 1$ : the coupling process is stable $\forall \alpha_{f} \geq 0$

- $B i^{(\Delta)}\left(1+\bar{D}_{f}\right) \leq 1$ : the coupling procedure exhibits the upper stability bound $\alpha_{f}^{\max }$

\subsection{Optimal procedure}

The optimal coefficient, $\alpha_{f}^{(o p t)}$, is given by

$$
\alpha_{f}^{(o p t)}=\frac{2 K_{s} K_{f}}{2 K_{s}-K_{f}\left(1+\bar{D}_{f}\right)}=\frac{2 K_{f}}{2-B i^{(\Delta)}\left(1+\bar{D}_{f}\right)}
$$

Note that this time, in the Neumann-Robin procedure, a positive coefficient $\alpha_{f}^{(o p t)}$ can only be defined conditionally if

$$
B i^{(\Delta)}\left(1+\bar{D}_{f}\right) \leq 2
$$

Thus, in the framework of the Neumann-Robin procedure, three zones can be identified. The first zone exhibits an upper stability limit defined by (20). In this zone, there is an optimal coefficient $\alpha_{f}^{(\text {opt })}$ for which the modulus of the amplification factor attains an absolute minimum.

The second zone is very narrow. It also presents an optimal coefficient, but in contrast to the previous case, there is no stability bound.

The third zone is also unconditionally stable, but no optimal coefficient can be defined. That is to say the minimum of the amplification factor is obtained for $\alpha_{f}=\infty$ (Neumann-Dirichlet procedure-heat flux imposed on the fluid side and temperature imposed on the solid side). The amplification factor is here a monotone function.

These three zones are clearly illustrated by the three curves depicted in Figure 5. 


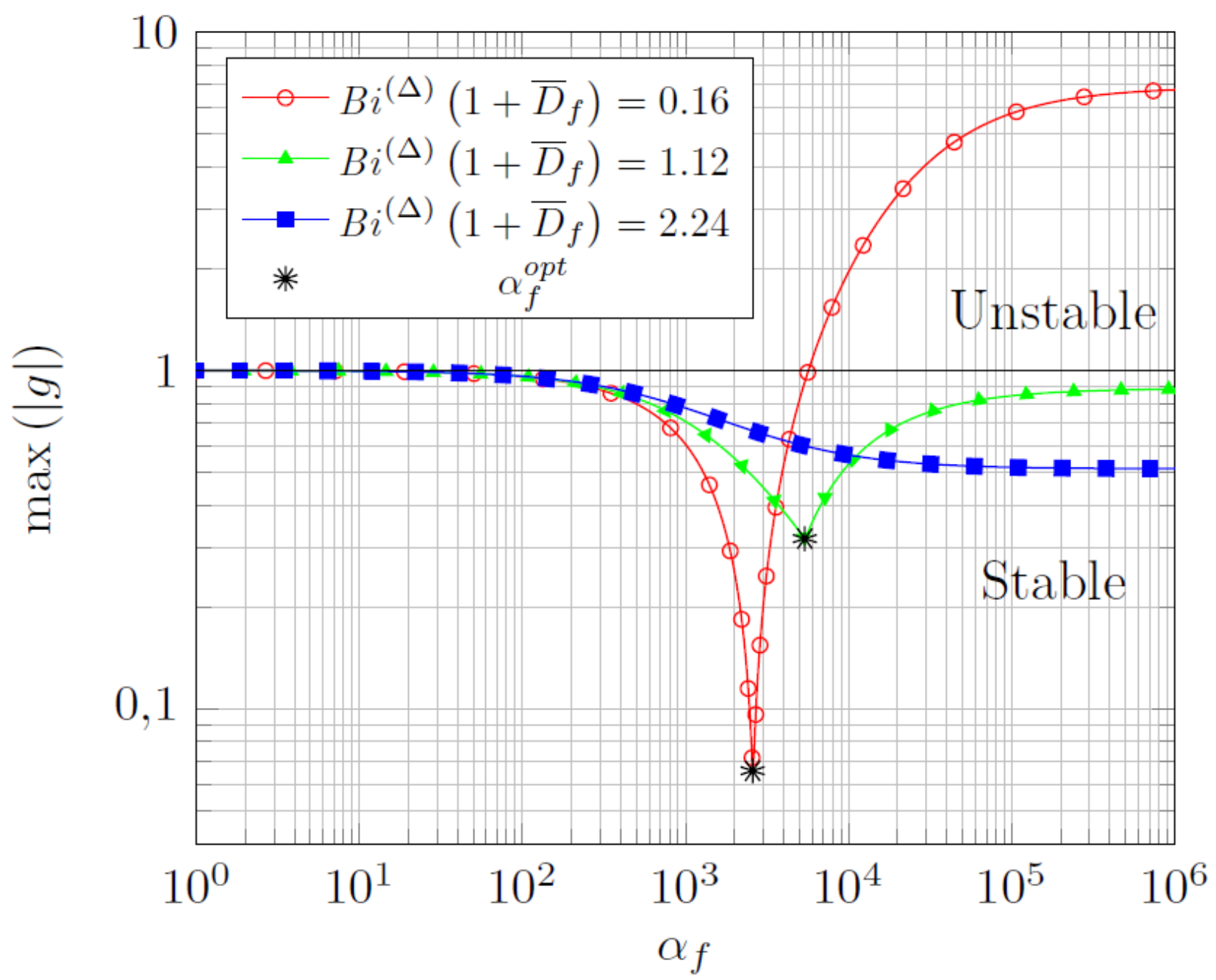

Figure 5. Temporal amplification factor of Neumann-Robin condition for three mesh Biot numbers Red curve : conditionally stable (low F-S interaction) and optimal coefficient Green curve : unconditionally stable (moderate F-S interaction) and optimal coefficient Blue curve : unconditionally stable (high F-S interaction) and no optimal coefficient

\subsection{Summary}

The general behavior of the Neumann-Robin coupling procedure for steady CHT is illustrated, in terms of $\alpha_{f}$ in Table 2. At low thermal F-S interaction, the amplification factor is partly outside the stability domain (first row in Table 2) and completely inside the stability domain for higher thermal interactions (second row in Table 2). These two functions present an optimal coefficient. On the contrary, for very high F-S interactions, the curve is totally inside the stability domain and no optimal coefficient can be defined (third row in Table 2). 


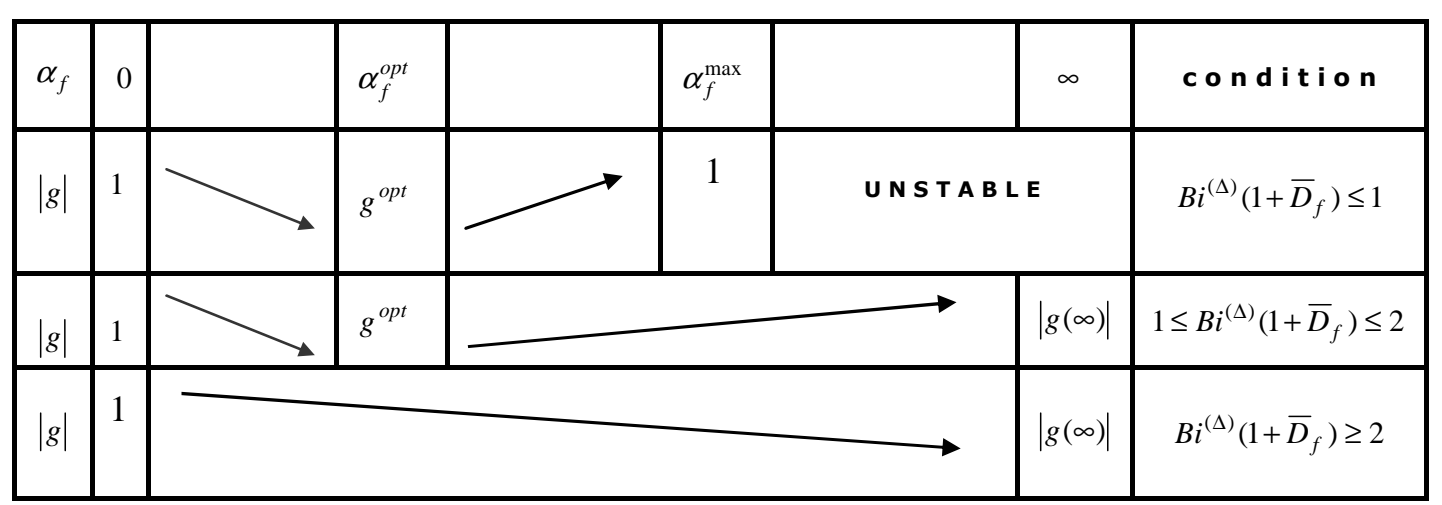

Table 2 : Numerical behavior of Neumann-Robin procedure $v s \alpha_{f}\left(\alpha_{s}=0 ; \alpha_{f} \geq 0\right)$

To the best of our knowledge, the mathematical expression of the upper stability bound, the expression of the so-called optimal coefficient, as well as the numerical properties of the NeumannRobin transmission conditions have never been published before.

\subsection{Stabilizing effect of the Fourier number}

As discussed before, the two interface conditions considered in this paper may be regarded as two complementary conditions. The first is unconditionally stable at small numerical Biot numbers whereas the second is unconditionally stable at large numerical Biot numbers.

\subsection{Overlapping zone}

The two interface conditions exhibit an overlapping zone in which both may be considered stable. Given the stability limits presented in the preceding sections, there is a visibly overlapping zone where both procedures are unconditionally stable. This zone is defined by

$$
\frac{1}{1+\bar{D}_{f}} \leq B i^{(\Delta)} \leq \frac{1}{1-\bar{D}_{f}}
$$

This overlapping zone becomes narrower as the normalized Fourier number $\bar{D}_{f}$ gets smaller. Conversely, this zone becomes significantly extended for large Fourier numbers.

\section{SUMMARY}

It is often argued that, for stability reasons, the best choice in a partitioned CHT approach is the classical Dirichlet-Neumann scheme, i.e. where the fluid is supplemented with the Dirichlet condition (temperature) on the fluid side. Nevertheless, the theoretical approach in Sections $3 \& 4$ shows that the situation is not quite so simple or straightforward, and that there are even arguments against this statement. A short summary is provided below.

Two different cases must be considered.

\section{(1) Fluid transient effects are predominant}

This is the most general situation and no assumptions on the temporal advancement of the fluid domain are required. Special attention must thus be given to condition (15) 
$\left(B i_{v}=B i^{(\Delta)}\left(1-\bar{D}_{f}\right) \leq 1\right)$. If this condition holds, the "transient" thermal resistance of the fluid domain at the shared interface is greater than the resistance offered by the whole solid domain. A Dirichlet condition on the fluid side is therefore appropriate.

If, in contrast, condition (16) holds $\left(B i_{v}=B i^{(\Delta)}\left(1-\bar{D}_{f}\right) \geq 1\right)$, and in extreme cases where $B i_{v} \gg>1$, then it is implied either that the solid thermal gradients are not negligible or that the thermal fluid conductance is larger than that of the solid. In this case, a Dirichlet condition imposed on the fluid is unlikely to provide the most efficient solution, even though the optimal coefficient is able theoretically to stabilize and optimize the procedure. The test case presented in Section 6 will serve as an illustration.

It should however be underlined that there is a powerful argument in favour of the Dirichlet condition. Indeed, the term $\left(1-\bar{D}_{f}\right)$ may become as small as necessary and thus the unconditional stability condition $B i^{(\Delta)}\left(1-\bar{D}_{f}\right) \leq 1$ ( $2^{\text {nd }}$ row of Table 1$)$ can always be satisfied. As a result, the stability bound can theoretically be removed by an appropriate choice of $\bar{D}_{f}$, i.e. by an appropriate choice of the time step. Conversely, in the condition $B i^{(\Delta)}\left(1+\bar{D}_{f}\right) \leq 1$ ( $1^{\text {st }}$ row in Table 2$)$, the term $\left(1+\bar{D}_{f}\right)$ has no stabilizing effect.

Nevertheless, we must not forget that for large $B i^{(\Delta)}$ and for a given (small) time step, the Dirichlet condition on the fluid side is prone to instability (1st row of Table 1), i.e. the part of the left-half line in Figure 3 is greater than unity.

\section{(2) Steady-like fluid solutions}

The heat transfer coefficient $h$ is often used [36] [41] [42] in this situation, when the fluid domain is updated only after a sufficient level of advancement in time. The fluid solution is not necessarily fully converged. The implementation of this heat transfer coefficient can only be defined after a sufficient number of fluid iterations have been carried out between two coupling instants. On this basis, a steady-like fluid state can be considered ( $\bar{D}_{f} \approx 0$ ). From (12) the stability condition of the steady F-S equilibrium is thus given by

$$
\alpha_{f}>\frac{1}{2}\left(h-K_{s}\right)=\frac{K_{s}}{2}(B i-1)
$$

where $B i$ is the conventional Biot number (18).

Consequently, the coefficient $\alpha_{f}=h$ satisfies the stability condition (24), thereby providing a better understanding of why this choice can be appropriate (but not optimal). Keep in mind, however, that this procedure is valid only if the fluid transient thermal effect becomes negligible.

Using the conventional Biot number instead of the mesh Biot number, Eq. (24) reveals two types of thermal interaction at steady state :

- $B i<1$ : The stability condition (24) is automatically fulfilled for any positive coupling coefficient $\alpha_{f}$. 
- $B i>1$ : The stability condition is given by (24).

This stability condition appears only when a temperature is imposed on the fluid side. At $B i>1$, the CHT procedure becomes unconditionally stable when the heat flux (Neumann condition) is imposed (no stability bound as shown in the $3^{\text {rd }}$ row of Table 2 ).

However we would like to point out once again that, although the conditions based on the conventional Biot number are theoretically valid to determine the stability of the fluid-solid equilibrium at steady-state, they are not appropriate to characterize the stability properties during a coupling process when fluid transient effects are not negligible. In addition, choosing a relevant reference temperature is a delicate task. As a result, the choice of the heat transfer coefficient, $h$, is questionable.

Finally, it is worth noting that these conclusions are valid for a steady CHT procedure in which a transient (or steady-like) fluid solution is used. For other coupled aero-thermal problems, stability considerations must be considered specifically [33].

\section{TEST CASE}

\subsection{CHT tests}

In the following, computing results are presented to illustrate the importance of the interface treatment in CHT problems. This has been studied employing the Dirichlet-Robin conditions with an emphasis on the optimal treatment. Then, Neumann conditions were employed on the fluid side in accordance with the conditions highlighted in this paper.

\subsection{Numerical tools}

The solid solver: The computer code in the solid, called Zébulon [43], is a three-dimensional finite-element code. It is an advanced object-parallel code for structural mechanics with many nonlinear solution capabilities. Zébulon can solve many types of heat transfer problems and particularly those in which the temperature field is calculated with no consideration of the stress/deformation in the overall structure. Only this solver will be employed in the study presented in this paper.

The fluid solver : elsA is a multi-application CFD simulation platform dealing with internal and external aerodynamics from the low subsonic to the high supersonic flow regime [44][45][46]. The compressible 3-D Reynolds averaged Navier-Stokes equations for arbitrary moving bodies are solved by a cell centered finite-volume method with second order upwind or central space discretization containing scalar or matrix artificial dissipation on multi-block structured meshes. A high flexibility in the multi-block approach is achieved in elsA through the patched grid, hierarchical mesh refinement and Chimera techniques. The discrete equations are integrated either by multistage Runge-Kutta schemes with implicit residual smoothing, or (which in general leads to a better efficiency) by backward Euler integration with implicit LU schemes. A large variety of turbulence models are available, ranging from eddy viscosity to full differential Reynolds stress models, which include options for Detached Eddy Simulation (DES) and Large Eddy Simulation (LES). Various transition models are also available for complex geometry configurations. The elsA software package includes an important module for dealing with aeroelasticity. Also, a module dealing with calculation of gradients by linearized equation or by adjoint solver techniques is very useful for aerodynamic optimization. 
The coupling library : The coupling between these two computer codes has been carried out through the coupling library CWIPI [47], developed by ONERA. CWIPI (Coupling With Interpolation Parallel Interface) is a library that makes it possible to couple an arbitrary number of parallel codes with MPI communications. Coupling is made through an exchange zone that can be discretized in a different way on any coupled code. Linear, surface or volume couplings are available. CWIPI takes into account all types of geometrical elements (polygon, polyhedral); there is no requirement about the mesh nature.

\subsection{Test case}

The interface treatments presented so far are now applied to an academic simple 2D test case. We consider the problem of convective heat transfer over, and the conduction heat transfer within, a flat plate. The geometry and boundary conditions are illustrated in figure 6 .

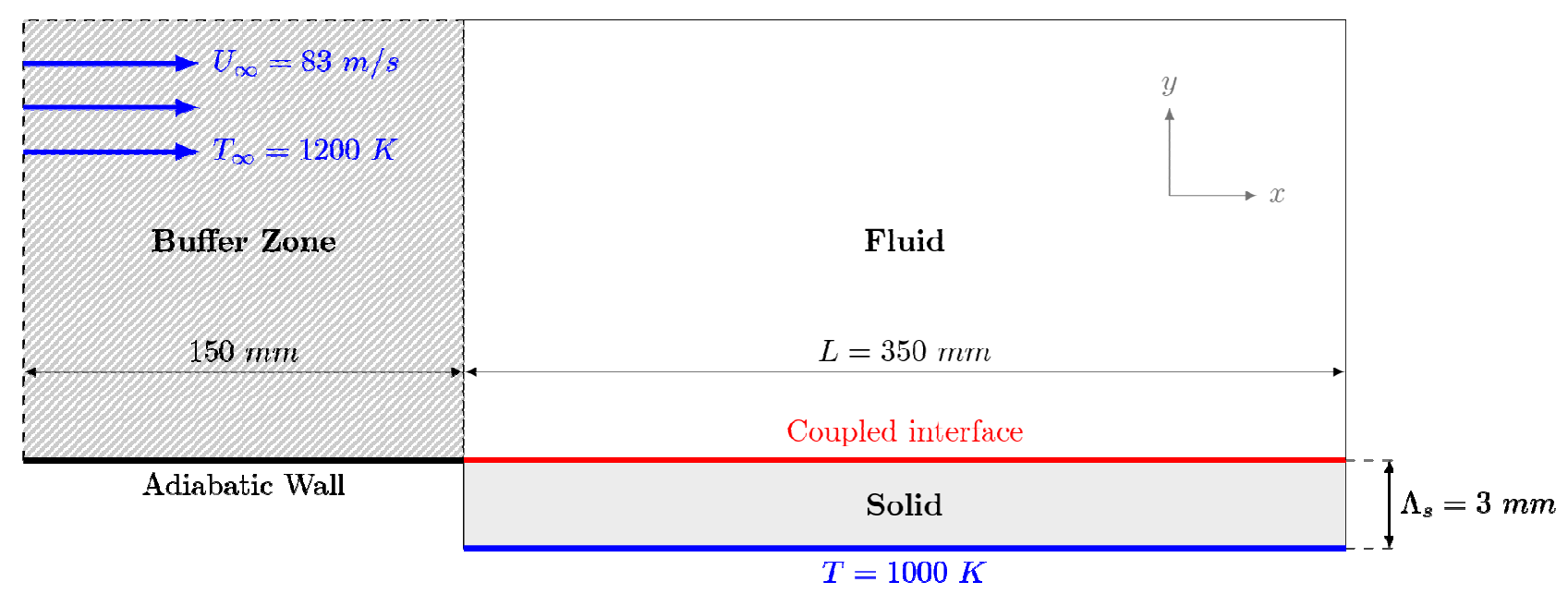

Figure 6. $2 \mathrm{D}$ test case

\subsubsection{Fluid domain}

The fluid domain is a rectangular channel $500 \mathrm{~mm}$ long with a symmetry boundary condition on the upper side. In the $z$-direction, there are no gradients. The interaction between the inlet and the viscous wall boundary conditions creates a singularity at the point where these two boundary conditions join. This singularity could generate numerical instabilities in the coupling process. Thus, a buffer zone with an adiabatic wall enables us to remove this singularity and to have a wellestablished fluid flow at the coupled interface. The presence of an adiabatic viscous wall induces a thermal leading edge at the flat plate.

Turbulent air flows from the inlet to interact with the upper wall of the solid plate (coupled interface), before exiting. A near wall well-refined mesh $\left(y^{+} \sim 1\right)$ was employed to correctly capture the flow boundary layer. That gives us the optimal calculation of the heat transfer. Most importantly, the convection contribution on the coupling process can be neglected (hypothesis of the 1D model), so no wall functions have been employed. Figure 7 shows a part of the fluid-solid mesh which is extremely refined at the interface $\left(\Delta y_{f}=5.4 \cdot 10^{-5} \mathrm{~m}\right)$.

The temperature of the fluid is $1200 \mathrm{~K}$, i.e. $200 \mathrm{~K}$ higher than the initial temperature of the flat plate. 


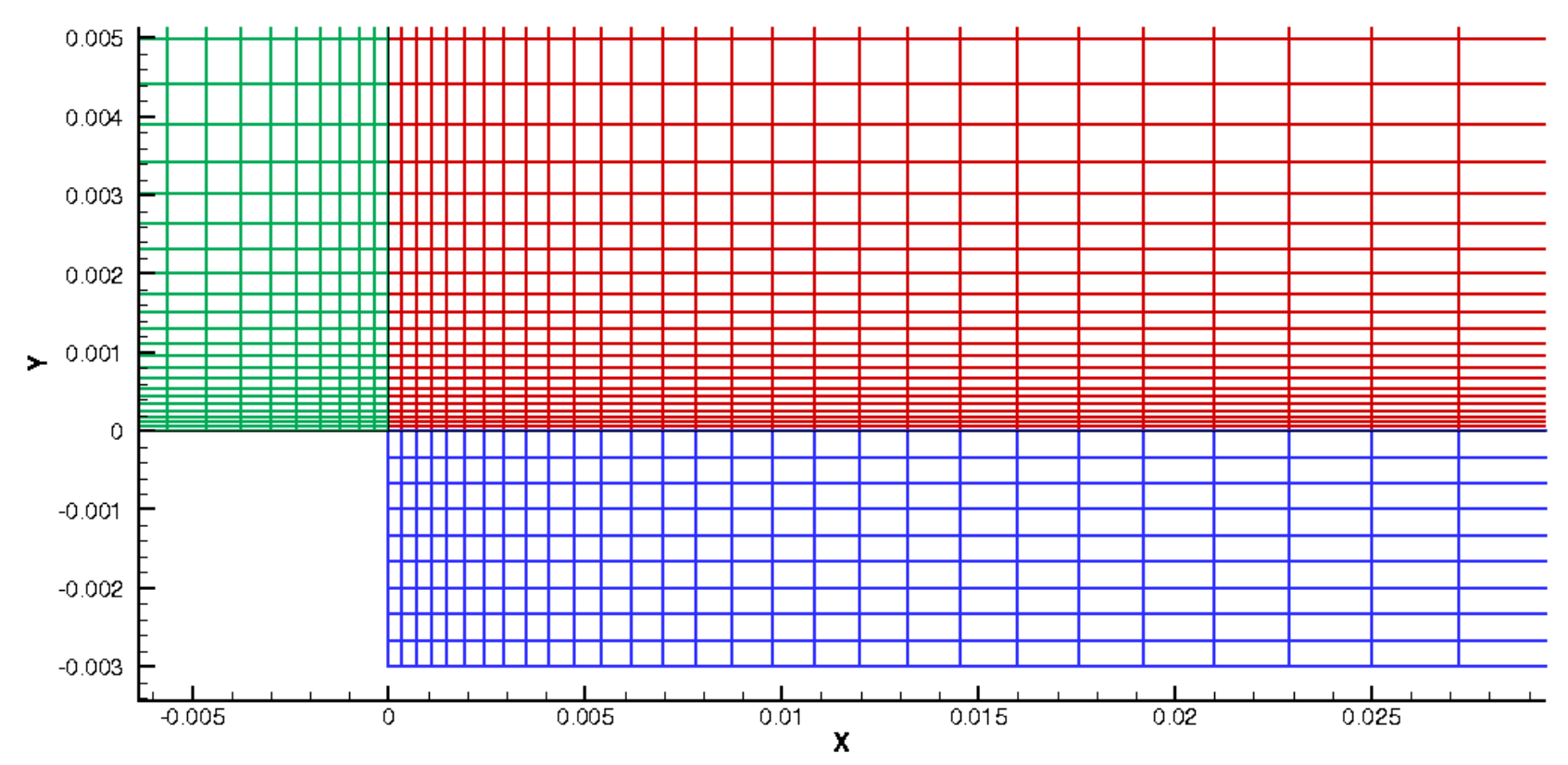

Figure 7. Fluid-solid mesh near the leading edge of the flat plate.

The red and blue meshes are the coupled fluid and solid meshes respectively. The green one is the buffer zone mesh.

The mesh illustrated in Figure 8 has been adapted to capture a fairly high exchange coefficient at the leading edge of the coupled interface. In fact a refined mesh in the $x$-direction near the leading edge gives a better estimation of the shear stresses in the leading edge (theoretically infinite).

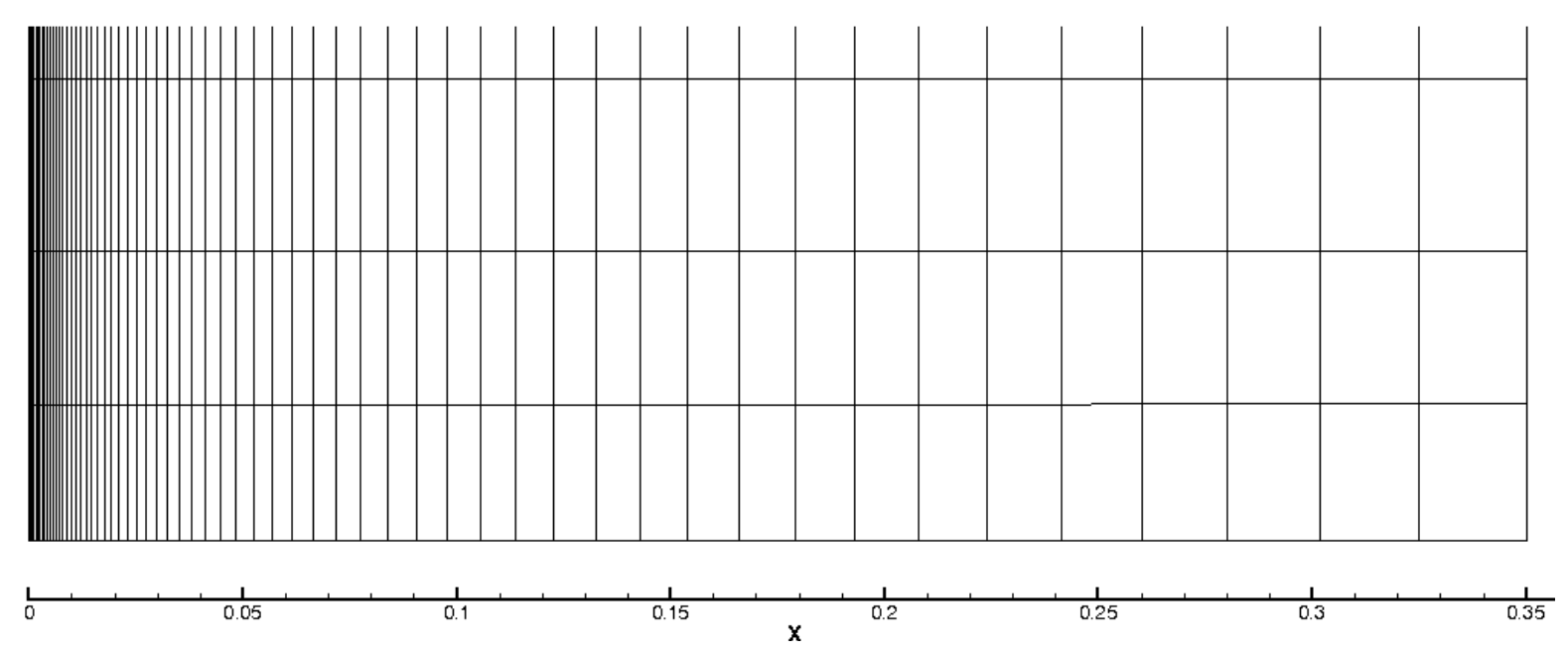

Figure 8. Refined fluid mesh at the Leading Edge

Figure 9 shows the heat transfer coefficient, $h$, along the flat plate obtained with the grid of Figure 8 . The refinement $\left(\Delta x_{f}=3 \cdot 3 \cdot 10^{-4} \mathrm{~m}\right)$ is sufficient at the leading edge to obtain a coefficient 
that varies from 3938 to 111 . This interval is large enough to test this coefficient on the following test cases.

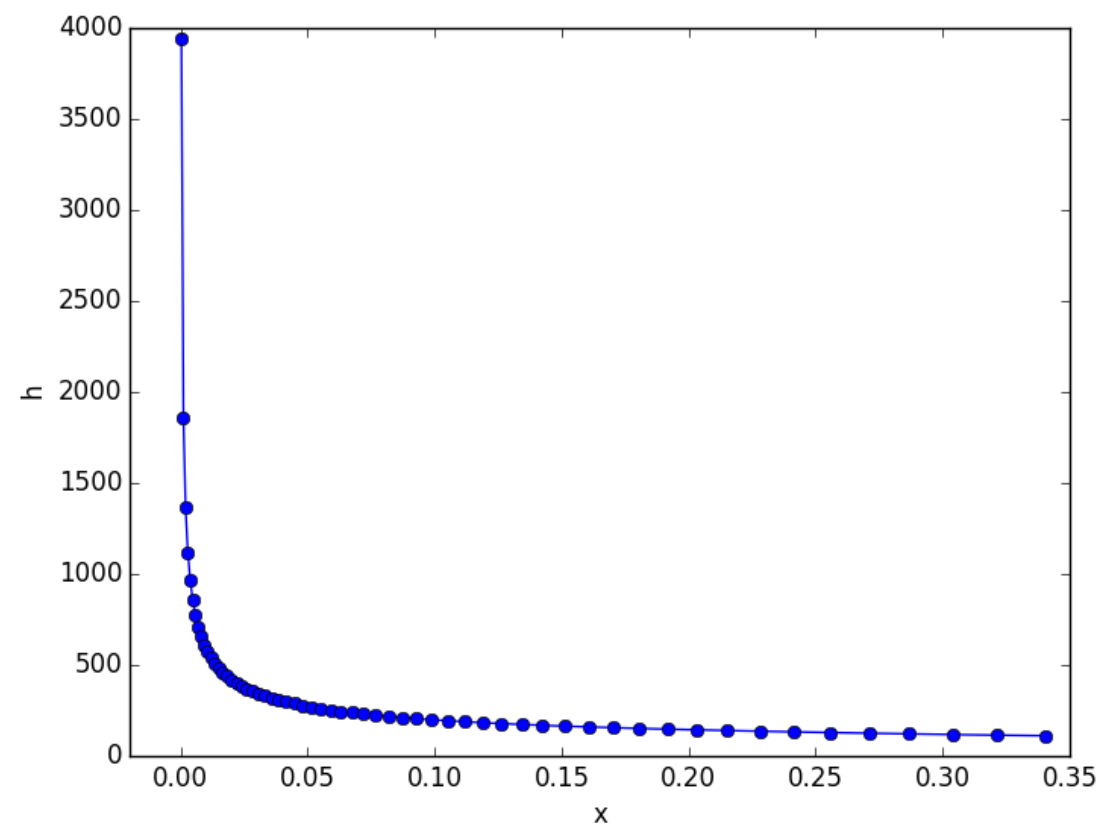

Figure 9. Heat transfer coefficient $h$, along the flat plate.

\subsubsection{Solid domain}

The solid domain is a rectangular flat plate, $3 \mathrm{~mm}$ thick and $350 \mathrm{~mm}$ long, i.e. the same sizes as the coupled fluid domain. The external faces of the solid plate are supposed to be adiabatic and a constant temperature $(1000 \mathrm{~K})$ is imposed on the lower side of the solid. The solid contains 10 mesh-points uniformly distributed in the $y$-direction (see Figure 7).

\subsubsection{Interface}

The coupling interface is $350 \mathrm{~mm}$ long and is composed of 60 elements. The fluid and the solid meshes are coincident at the interface (see Figure 7). This precaution was taken to avoid any spatial grid-to-grid interpolation error and to focus the numerical study on stability issues.

\subsubsection{Temporal parameters}

The coupled simulations were performed using the temporal parameters presented in Table 3.

\begin{tabular}{|c|c|c|c|c|}
\hline$\Delta t_{f}(\mathrm{~s})$ & $\Delta t_{c}(\mathrm{~s})$ & $D_{f}$ & $\bar{D}_{f}$ & $\left(1-\bar{D}_{f}\right)$ \\
\hline $3.10^{-5}$ & $6.10^{-4}$ & 400. & 0.93 & 0.07 \\
\hline
\end{tabular}

Table 3 : Temporal parameters

\subsubsection{Convergence Criterion}

The convergence criterion (error tolerance or stopping criterion) used in this paper is based on the infinity norm of the absolute interface temperature, defined by 


$$
\Delta T_{f}=\max _{i=1, N_{f}}\left|T_{i}^{n}-T_{i}^{n-1}\right|
$$

where $n$ is the coupling iteration and $i$ represents the spatial index along the fluid interface $\left(N_{f}\right.$ faces). We will consider a simulation to have converged if :

$$
\Delta T_{f}<\varepsilon \quad \text { with } \quad \varepsilon=10^{-3}
$$

\subsubsection{Solid thermal conductivity and thermal F-S interaction}

The previous sections have shown that the numerical interface methods are directly dependent on the numerical Biot number. As the interest here is on implementing adaptive local schemes, it is fundamental to cover a broad range of different Biot numbers and ideally within the same flow field.

In the next two sections, the behavior of the interface conditions is studied with different materials by varying the solid conduction coefficient $\lambda_{s}$, i.e. the mesh and numerical Biot number. The following test cases are classified in two separate categories in accordance with the theory presented in the previous sections :

(1) Weak/moderate thermal interaction

(2) Strong thermal interaction.

For the weak/moderate F-S interaction, the Dirichlet-Robin interface condition is well-suited. For higher interactions, another interface treatment using the Neumann-Dirichlet condition will also be considered. 


\section{Weak and Moderate Thermal Fluid-Structure Interaction}

\subsection{Amplification factors}

Weak thermal interactions are characterized by low values of the numerical Biot number. To determine this, we should look at the amplification factor shown in Figure 10.

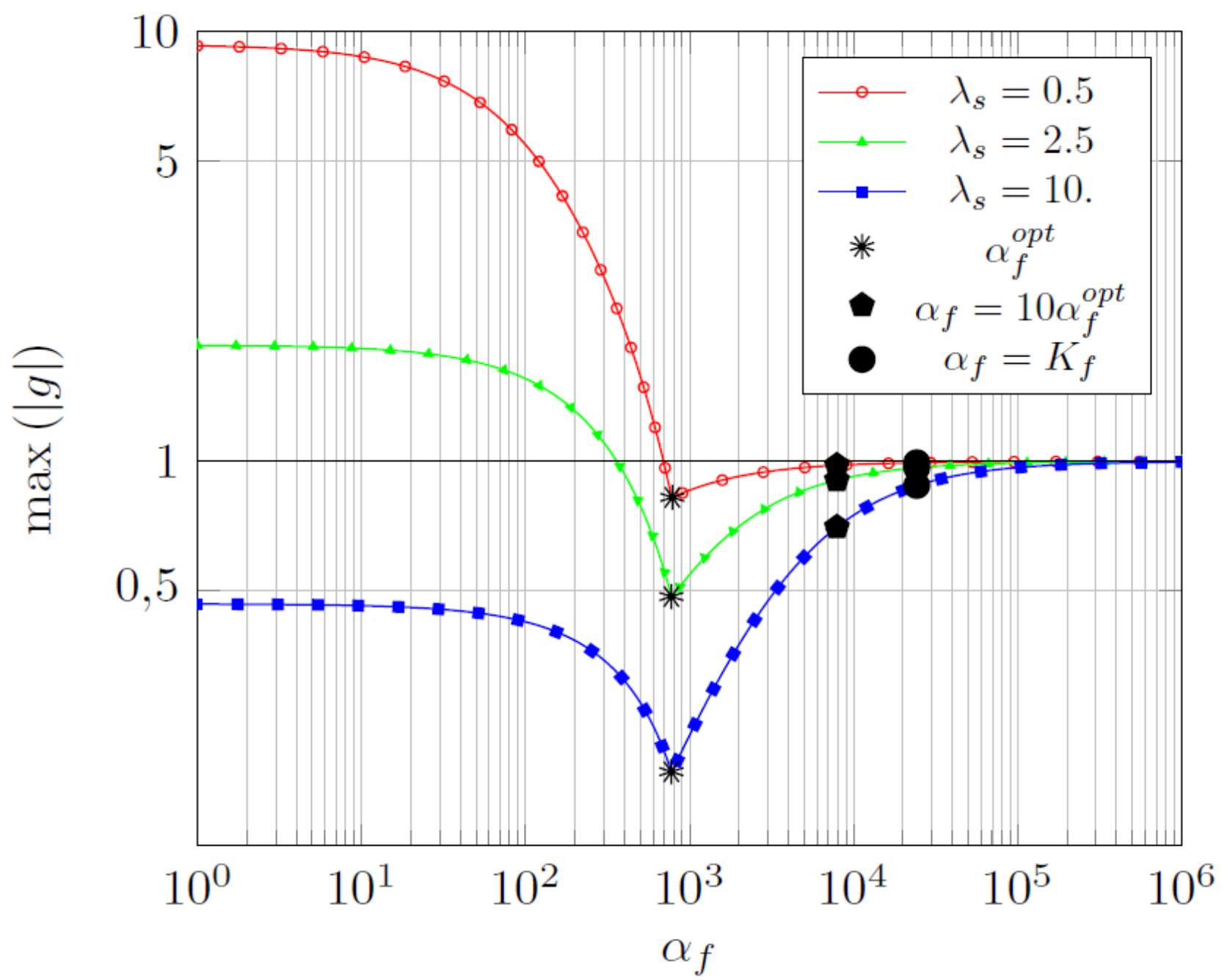

Figure 10. Amplification factor for three weak thermal interaction cases

The numerical Biot number represents the $y$-intercept $\left(\alpha_{f}=0\right)$. The higher this value, the greater the amplification factor. As can be seen, a weak thermal interaction case $\left(\lambda_{s}=10 . \mathrm{W} \cdot \mathrm{m}^{-1} \cdot \mathrm{K}^{-1}\right)$ along with two moderate ones $\left(\lambda_{s}=2.5\right.$ and $\left.\lambda_{s}=0.5\right)$ are considered. The curve for the weak thermal case is entirely within the stable area $(\max (|g|)<1)$ as the blue curve with square markers in Figure 10 shows. Thus, according to the model problem, it must be stable for all values of the $\alpha_{f}$ coefficient. For the other two cases, the left side of the curve is partly in the unstable zone and therefore should not be stable for low values of the coupling coefficient $\left(\alpha_{f}<\alpha_{f}^{\mathrm{min}}\right.$ ). 


\subsection{Dirichlet-Robin interface conditions}

Four coupling coefficients are used for the three test cases:

(0) $\alpha_{f}=0$ (Dirichlet - Neumann interface condition)

(1) $\alpha_{f}=\alpha_{f}^{(\text {opt })}$

(2) $\alpha_{f}=10 \cdot \alpha_{f}^{(o p t)}$

(3) $\alpha_{f}=K_{f}>\alpha_{f}^{(o p t)}$

In the case of a weak thermal interaction, the Dirichlet-Robin interface condition is clearly the most relevant as we have demonstrated in section 3. In Table 4, numerical numbers characterizing the thermal interaction are presented, namely the solid thermal conduction coefficient $\lambda_{s}$, the mesh Biot number $B i^{(4)}$ and the numerical Biot number $B i_{v}$ for the test case considered. In the same table, the number of coupling iterations needed to reach convergence is indicated.

\begin{tabular}{|c|c|c|c|c|c|c|c|}
\hline \multicolumn{4}{|c|}{ Parameters } & \multicolumn{4}{c|}{ Iterations to converge $\left(\varepsilon=10^{-3}\right)$} \\
\hline$\lambda_{s}$ & $K_{s}$ & $\begin{array}{c}B i^{(\Delta)} \\
\text { Eq.(13) }\end{array}$ & $\begin{array}{c}B i_{v} \\
\text { Eq.(14) }\end{array}$ & $\alpha_{f}=0$ & $\alpha_{f}=\alpha_{f}^{(o p t)}$ & $\alpha_{f}=10{ }^{*} \alpha_{f}^{(o p t)}$ & $\alpha_{f}=K_{f}$ \\
\hline 10. & 3333. & 6.6 & 0.5 & 62 & 62 & 65 & 76 \\
\hline 2.5 & 833. & 27.3 & 1.9 & 71 & 72 & 102 & 216 \\
\hline 0.5 & 166. & 141.5 & 9.4 & $\begin{array}{c}\text { Crash } \\
\text { at the } 4^{\text {th }} \text { it. }\end{array}$ & 84 & 250 & 591 \\
\hline
\end{tabular}

Table 4 : Numerical properties and convergence for weak \& moderate thermal interaction

The convergence history for the four values of the coupling coefficient, $\alpha_{f}$, is shown in Figures 11, 12 and 13, by plotting the interface temperature residuals, as a function of the coupling iteration. As might be anticipated, the convergence history largely depends on the value of the coupling coefficient. In weak thermal F-S interactions (Figure 11), there are no significant differences for low values of the coupling coefficient $\alpha_{f}$ and it can be seen that the trends marked by the two lines $\alpha_{f}=0$ and $\alpha_{f}=\alpha_{f}^{(o p t)}$ are roughly the same. For high values of the coupling parameter, convergence is a little slower, but at a tolerable level, even with $\alpha_{f}=K_{f}$ where a linear convergence is observed. 


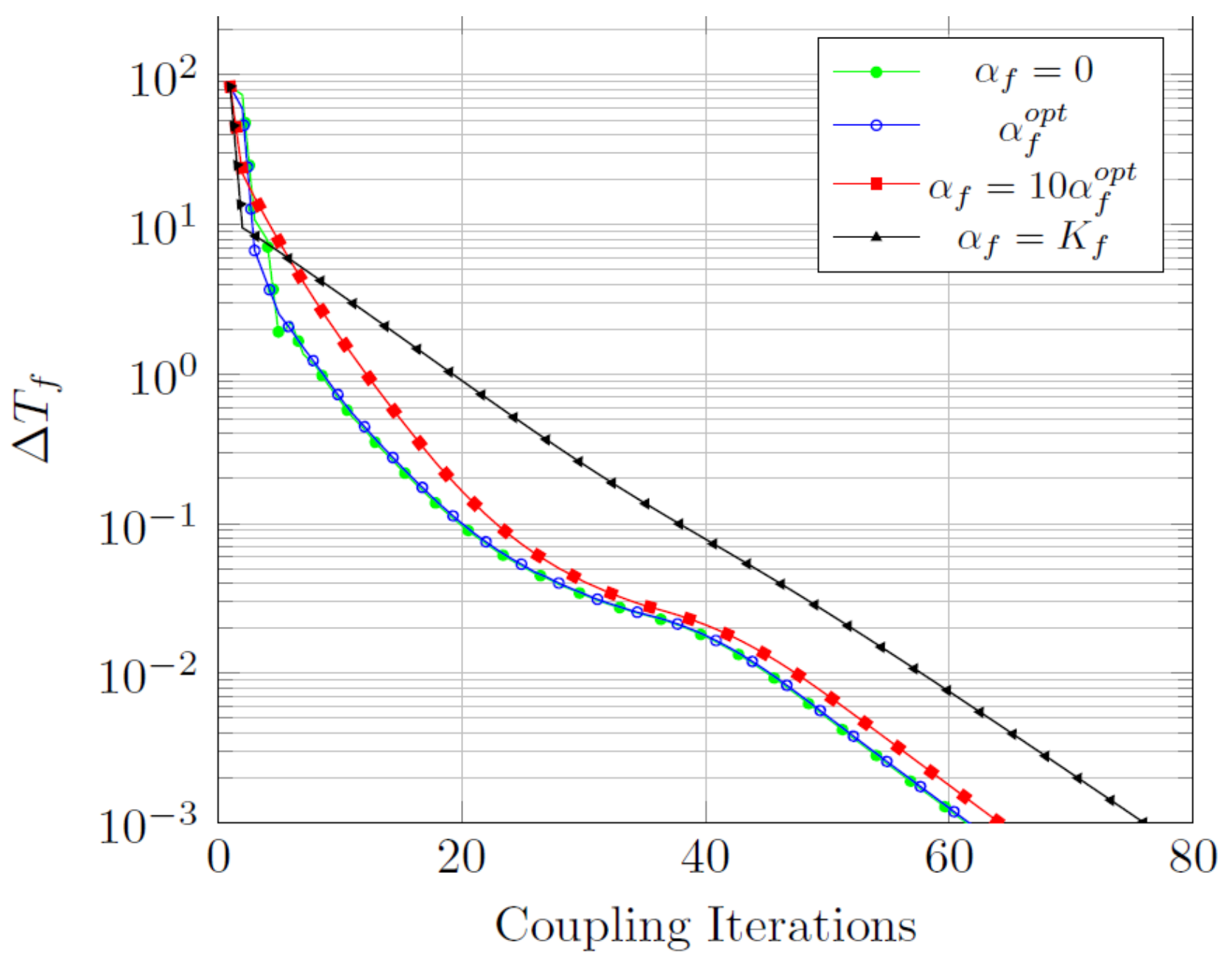

Figure 11. Convergence $v s$ coupling iterations $\left(\lambda_{\mathrm{s}}=10 \mathrm{~W} \cdot \mathrm{m}^{-1} \cdot \mathrm{K}^{-1}-B i_{v}=0.5\right)$

In the same manner as outlined above, in moderate thermal F-S interactions $\left(B i_{v}=1.9\right)$, there are no major differences for low values of the coupling coefficient $\alpha_{f}$ as shown in Figure 12. However, this time, note that the computation for $\alpha_{f}=0$ is supposed to be unstable according to the model problem (first row of Table 1). These discrepancies may be due to some stabilizing effect generated by the flow, not included in the theoretical model. It is necessary nevertheless to specify that even if convergence is rapidly reached, important oscillations of the interface temperature near the leading edge are observed during the initial phase of the coupling process for this value. On the contrary, for $\alpha_{f}=\alpha_{f}^{(o p t)}$, the temperature behavior is oscillation-free. For bigger values of $\alpha_{f}$, stable CHT computations are obtained, but they take a little longer. 


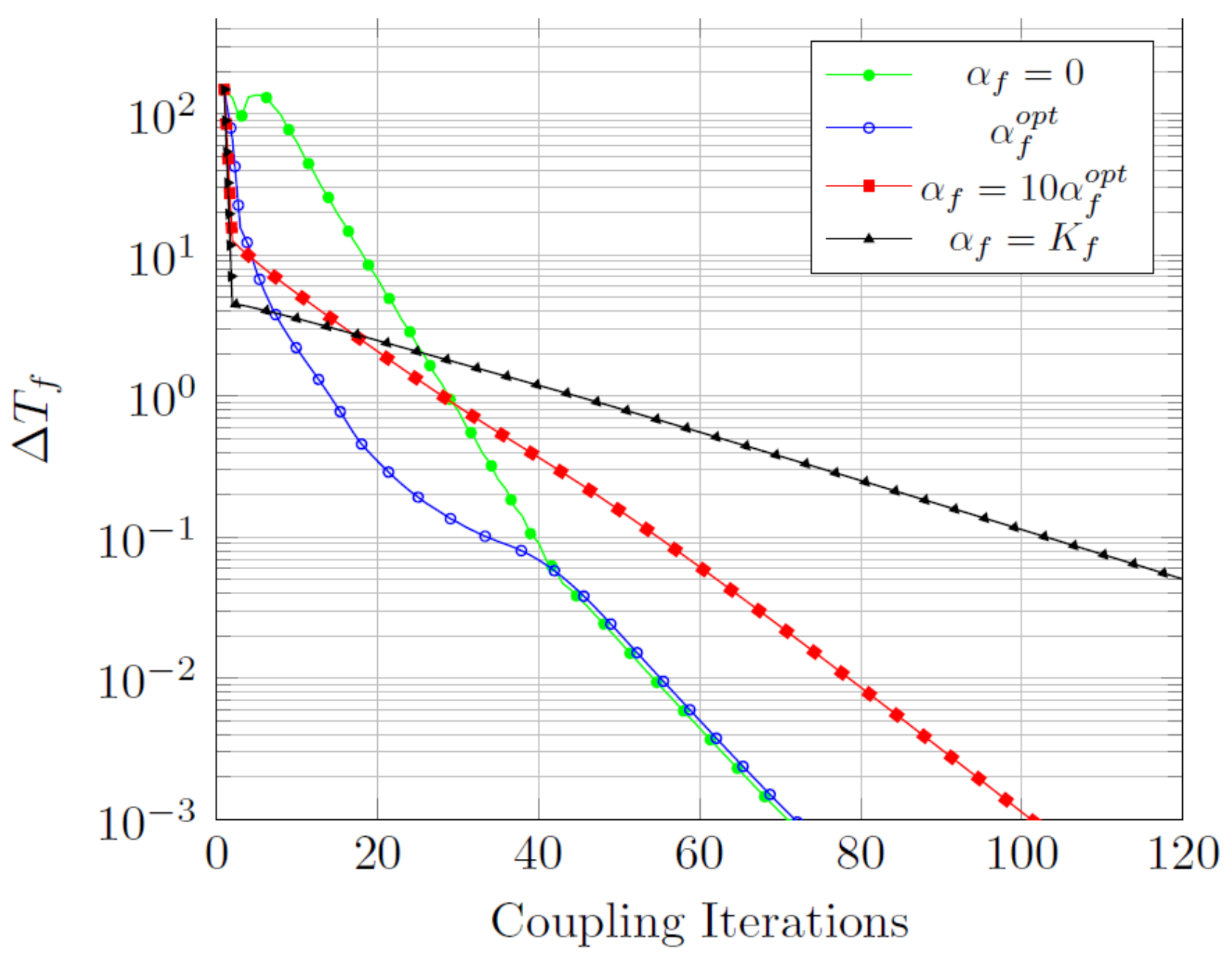

Figure 12. Convergence $v s$ coupling iterations $\left(\lambda_{\mathrm{s}}=2.5 \mathrm{~W} \cdot \mathrm{m}^{-1} \cdot \mathrm{K}^{-1}-B i_{v}=1.9\right)$

For a higher thermal F-S interaction $\left(B i_{v}=9.4\right)$, as expected, $\alpha_{f}=0$ leads to divergent computations after the $4^{\text {th }}$ coupling iteration (Figure 13). The other three coefficients result in stable computations. The fastest convergence is obtained for $\alpha_{f}=\alpha_{f}^{(o p t)}$. For $\alpha_{f}=K_{f}$, the coupling process is so slow that its convergence, at $\Delta T_{f}=10^{-3} \mathrm{~K}$, cannot be represented in this Figure. This indicates that the fluid-solid system tends to be "frozen" and thus, this over-stable solution becomes prohibitive. Clearly, the higher the thermal interaction, the more attention must be paid to the choice of the boundary conditions (at least with a Dirichlet condition imposed on the fluid side). 


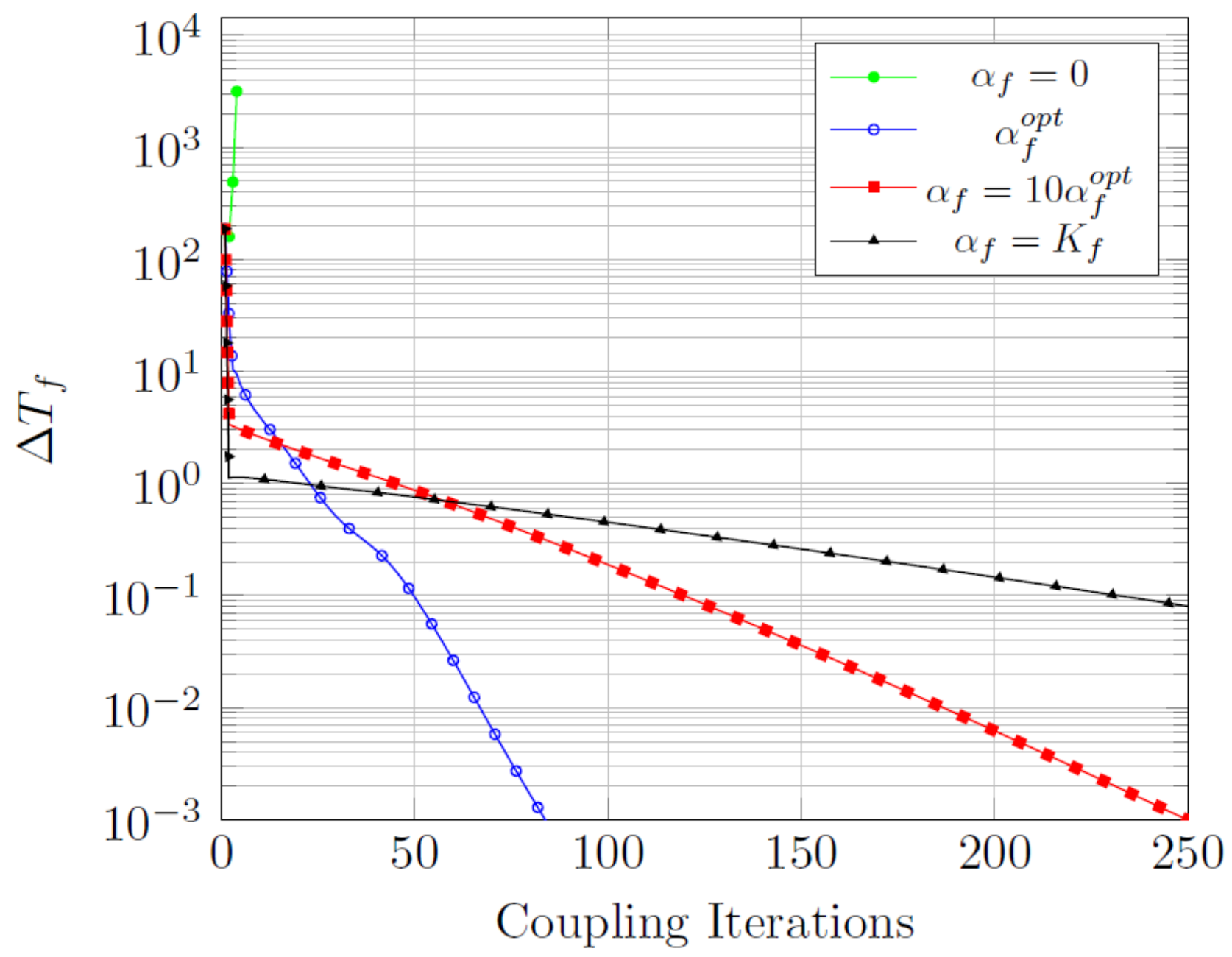

Figure 13. Convergence $v s$ coupling iterations $\left(\lambda_{\mathrm{s}}=0.5 \mathrm{~W} \cdot \mathrm{m}^{-1} \cdot \mathrm{K}^{-1}-B i_{v}=9.4\right)$

In summary, these convergence behaviors were largely predictable from the model problem. As expected, $\alpha_{f}^{(o p t)}$ appears to be the optimal choice, providing a monotonic, stable and fast convergence for all simulations.

\subsection{CHT simulation solution near the stability limit}

Now, emphasis is put on the last test case (with $\lambda_{s}=0.5 \mathrm{~W} \cdot \mathrm{m}^{-1} \cdot \mathrm{K}^{-1}$ and $B i_{v}=9.4$ ) to analyze the interface temperature close to the stability limit. Three points of the coupling interface have been selected, namely the leading edge, the midpoint and the trailing edge. Three values of $\alpha_{f}$ have been specifically chosen near the stability limit $\left(\alpha_{f}=380 ., \alpha_{f}=400\right.$. and $\alpha_{f}=\alpha_{f}^{(o p t)}$ ) to illustrate some specific and interesting trends. The location of these points along the temporal amplification factor curve is shown in Figure 14. 


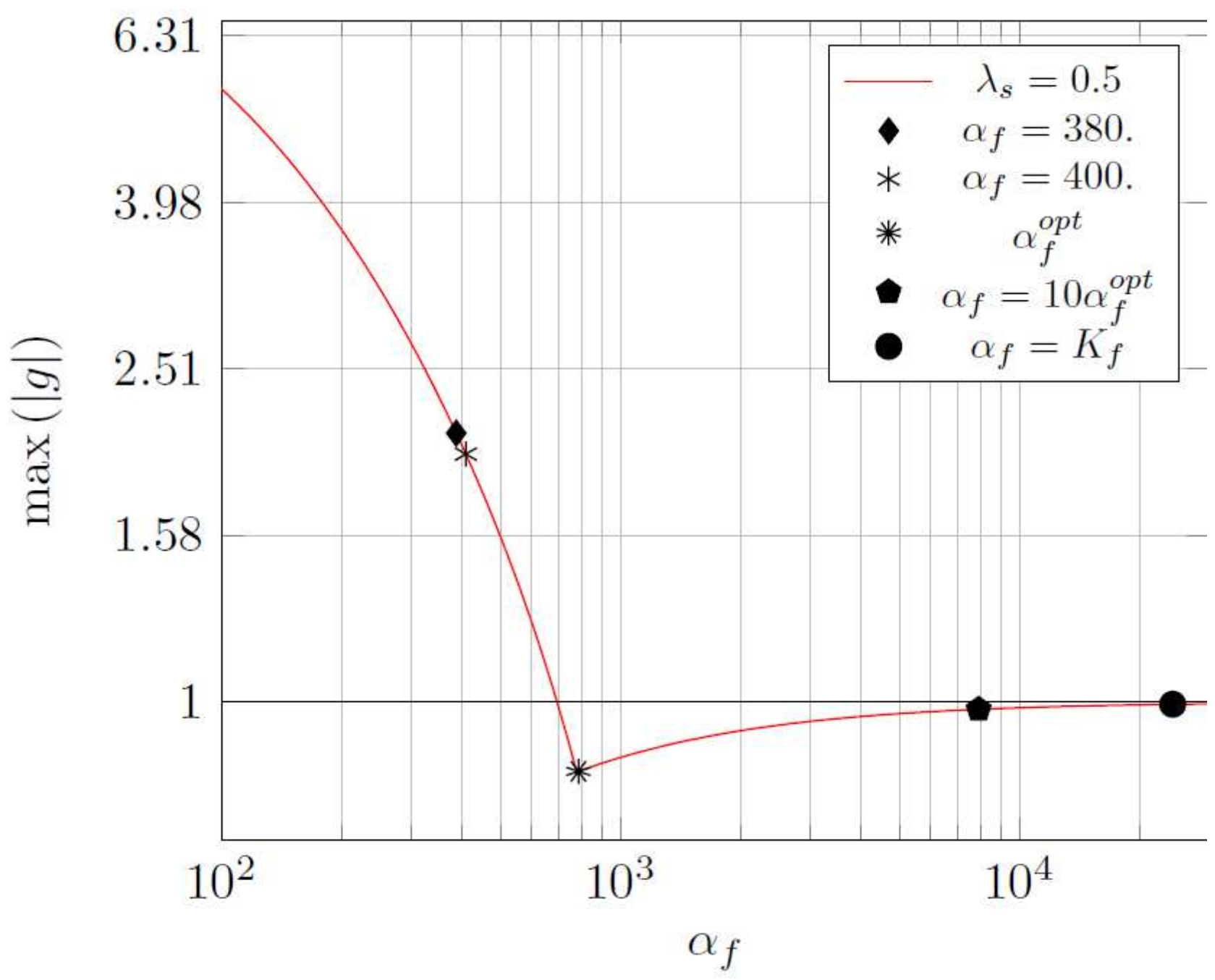

Figure 14. Amplification factor for $\lambda_{s}=0.5 \mathrm{~W} \cdot \mathrm{m}^{-1} \cdot \mathrm{K}^{-1}-B i_{v}=9.4$

First of all, it is interesting to observe, for $\alpha_{f}=380$, the very large oscillations (about $1000 \mathrm{~K}$ ) of the interface temperature at the leading edge, that do not decay (Figure 15). For the other two points of the interface, the computation converges rapidly. Clearly, this constant value of $\alpha_{f}$ is too small to stabilize the coupled process at the leading edge but sufficient for the other two points. 


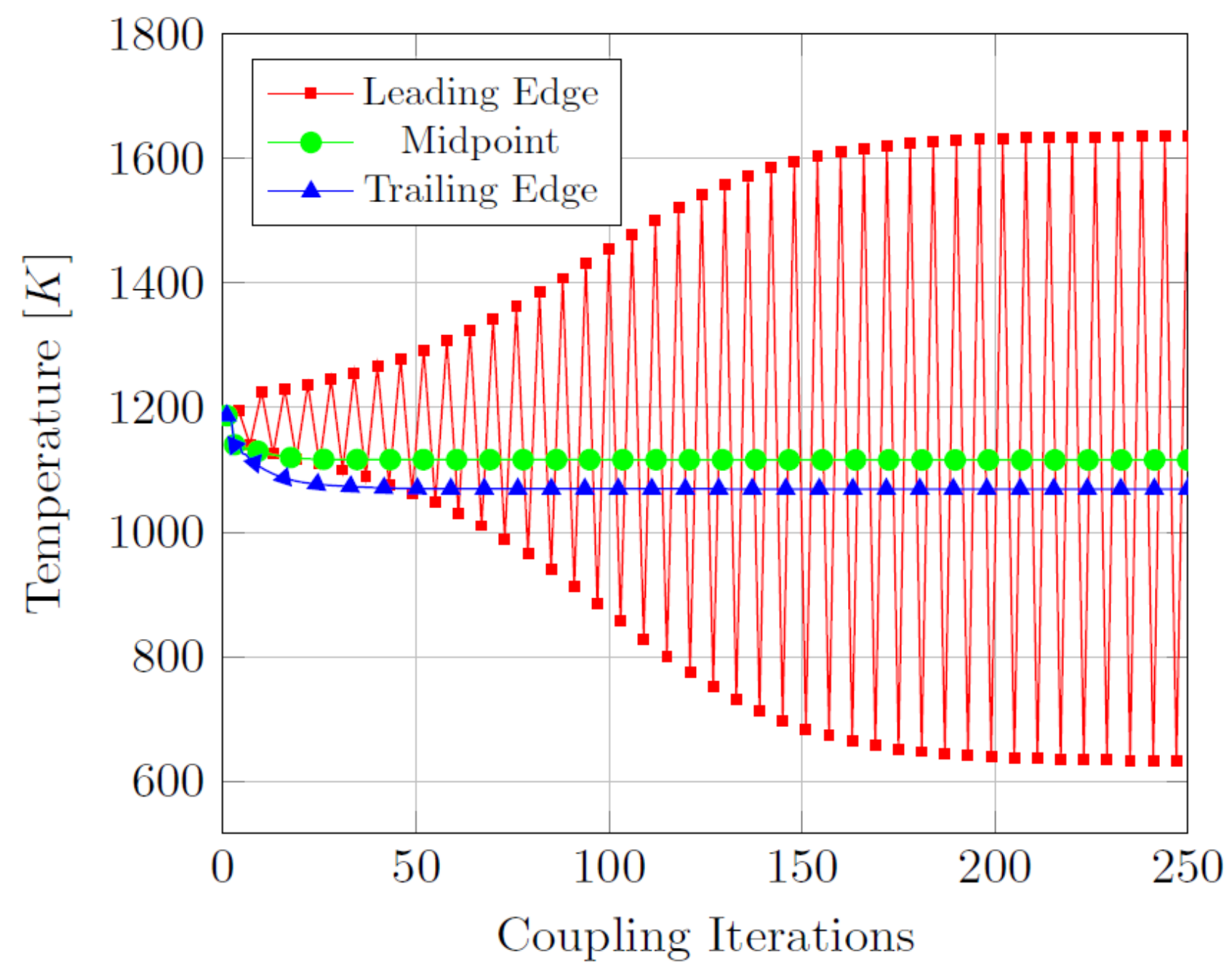

Figure 15. Temperature vs coupling iteration at the leading edge for $\alpha_{f}=380$. The dotted line indicates the temperature oscillation (one out of three points is shown) and the solid line highlights the bifurcation behavior.

By slightly increasing the coefficient ( $\alpha_{f}=400$.), the temperature oscillations at the leading edge are slowly damped (Figure 16). This is a good example of the need of a local coupling coefficient. 


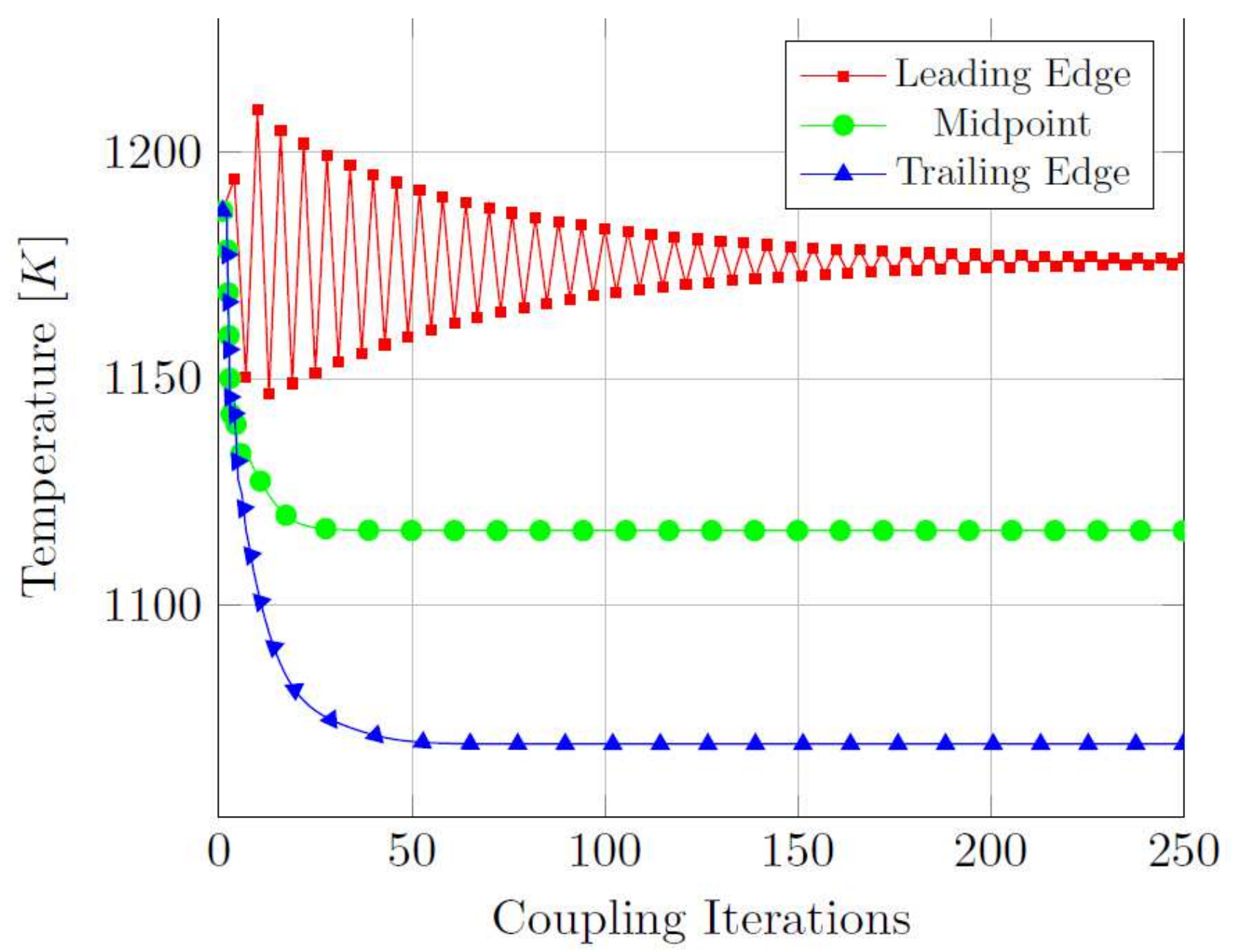

Figure 16. Temperature $v s$ coupling iterations for $\alpha_{f}=400$. (one out of three points is shown).

Interesting results have been found when $\alpha_{f}$ is taken as the heat transfer coefficient $h$ (Figure 17). This is a common practice in the literature. 


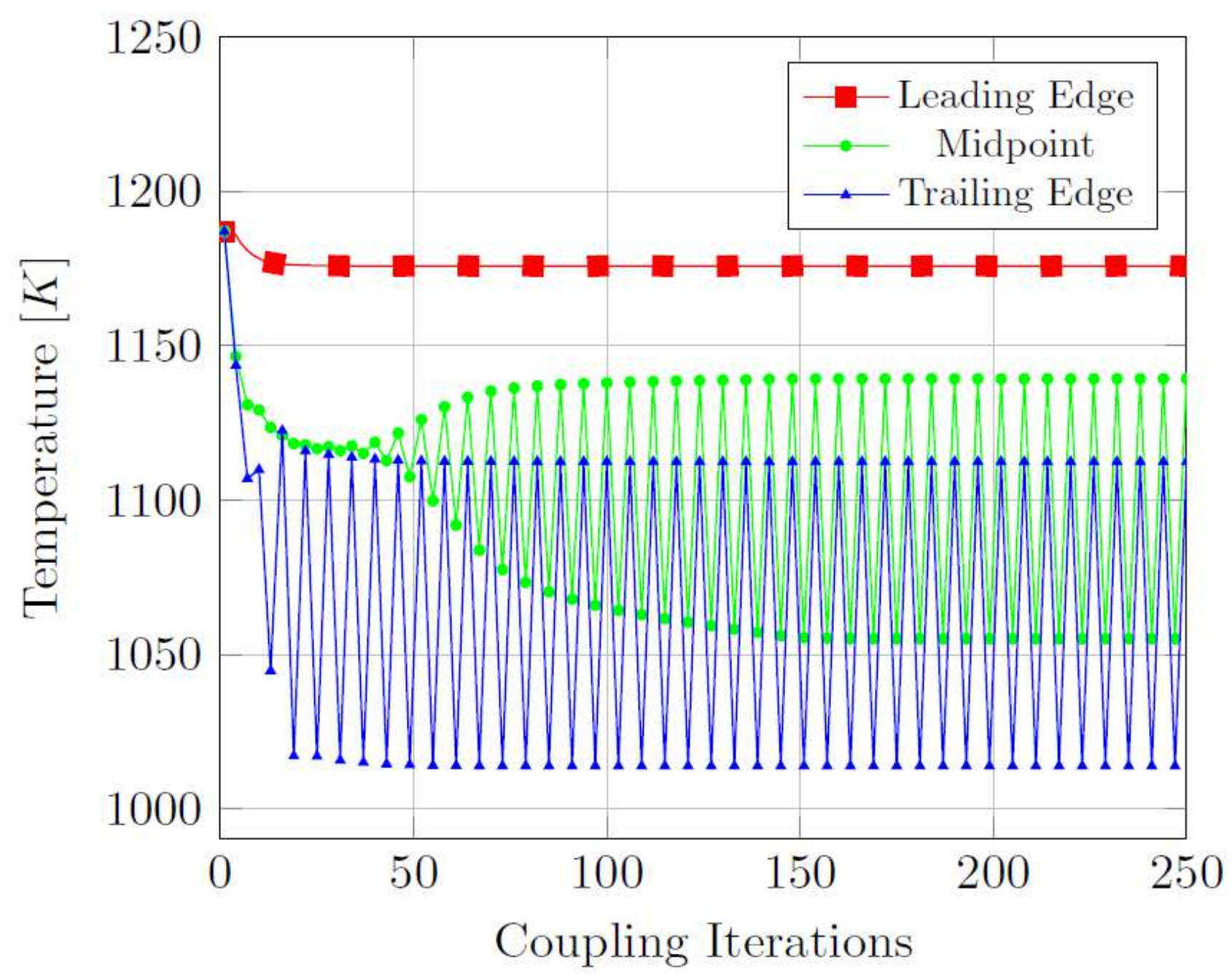

Figure 17. Temperature $v s$ coupling iterations for $\alpha=h(x)$ (a point on three is shown)

The heat transfer coefficient $h$ was calculated by:

$$
\alpha_{f}=h_{i}^{n}=\frac{q_{i}^{n}}{T_{i}^{n}-T_{r e f}}
$$

where the $i$-index indicates the point on the coupling surface and the $n$-index the current coupling iteration. $T_{r e f}$ is the reference temperature (we take it as the temperature of the incoming flow). In this case the $\alpha_{f}$ coefficient is local. The resulting $h$ evolution is similar to that shown in Figure 9. We can see that the leading edge area can be stabilized easily. However, it is not at all the case for the other two points located downstream where large oscillations can be observed. Indeed, at the leading edge, the heat transfer coefficient $h$ is very large (see Figure 9) and thus located on the right-hand side of the amplification curve, i.e. in the stable zone (see red curve in Figure 10). Then, it decreases sharply and the resulting coefficient has a too small stabilizing effect (value located on the left-hand side of the amplification factor curve). This illustrates unambiguously that the "convective" heat transfer coefficient is unsuitable for stabilizing the F-S diffusion interaction. 
The only way of preventing severe numerical problems in heterogeneous surfaces is to implement adaptive coefficients reflecting local coupling conditions. For instance, using the optimal coefficient $\alpha_{f}^{(o p t)}$, the temperature over the whole surface is stable and fast-converging as shown in Figure 18.

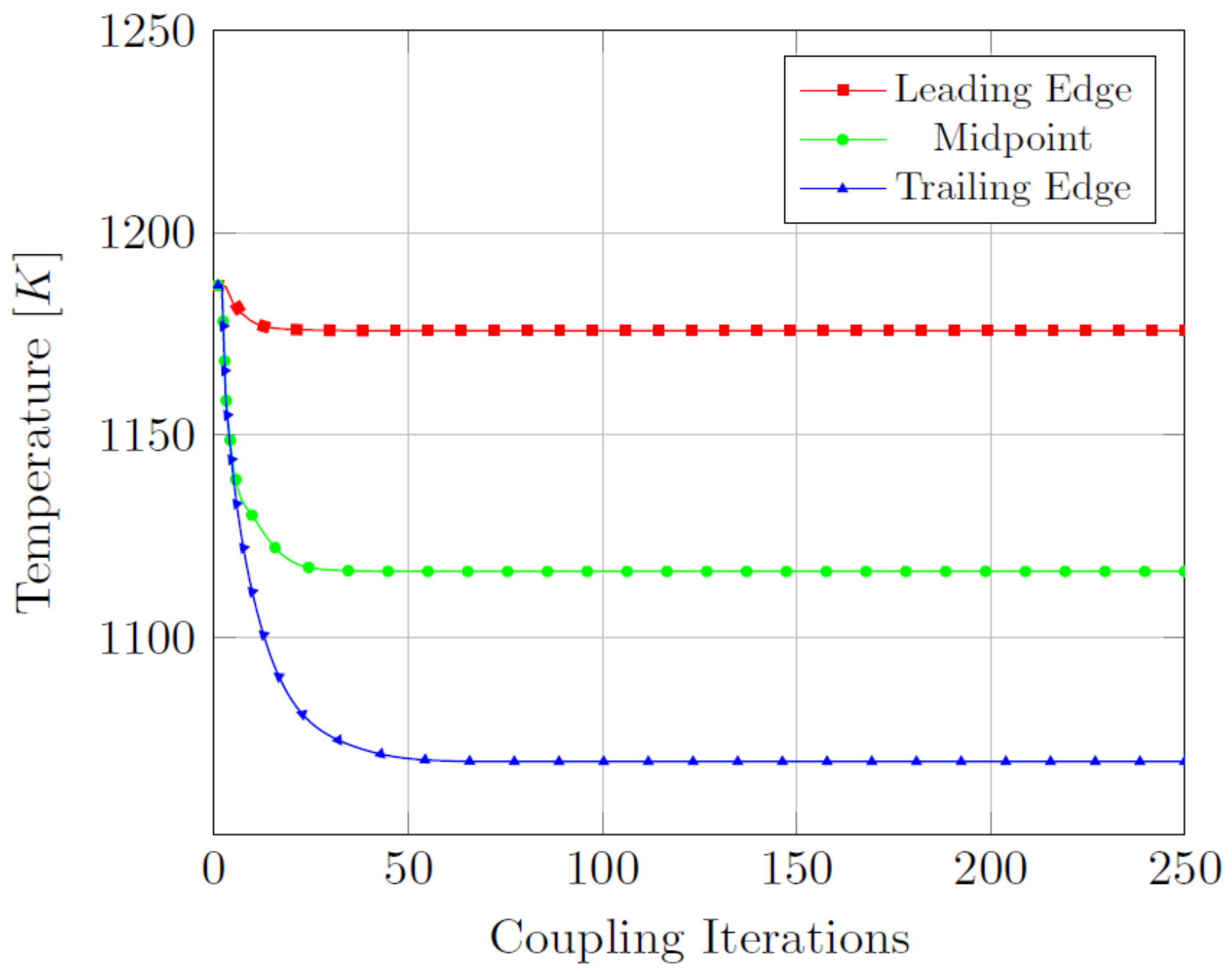

Figure 18. Temperature $v s$ coupling iterations for $\alpha_{f}=\alpha_{f}^{(o p t)}$

\subsection{Use of $\alpha_{f}=h$ near convergence}

In section 5 it was suggested that the heat transfer coefficient $\left(\alpha_{f}=h\right)$ could be used after a sufficient level of advancement in time. In other words, when the convective heat transfer is close to equilibrium with the solid conduction, but not perfectly converged. 


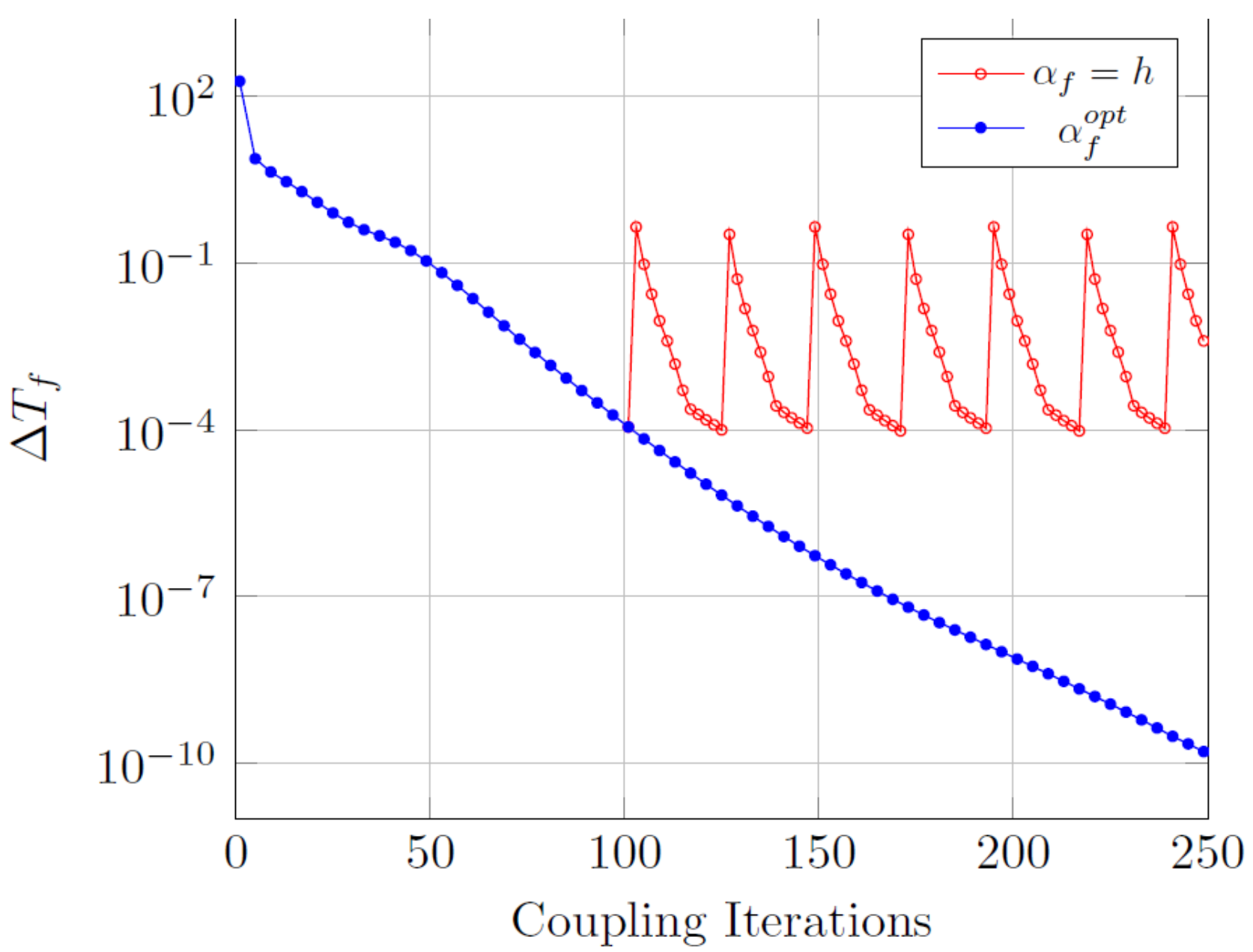

Figure 19. Convergence history of $\alpha_{f}=h$ from $\Delta T_{f}=10^{-4}$

It was therefore decided to implement the optimal coefficient $\alpha_{f}^{(\text {opt })}$ before switching to the condition $\alpha_{f}=h$ at a convergence level of $\varepsilon=10^{-4}$ (see Eq.(26)). Large and persistent oscillations suddenly occur, which means that the convective coefficient is not a relevant choice in this case (see Figure 19). As mentioned above, this coefficient produces under-relaxed solutions at the trailing edge, and this is confirmed even when the CHT solution is close to steady-state, i.e. quite converged. Furthermore, implementing a convective coefficient raises a fundamental issue, which is the choice of a relevant reference temperature.

\subsection{Comparison in performance between optimal CHT and CFD only}

Figure 20 shows the convergence history (residual of the mass density) between the CHT computation and a CFD only, with a given temperature prescribed at the interface. 


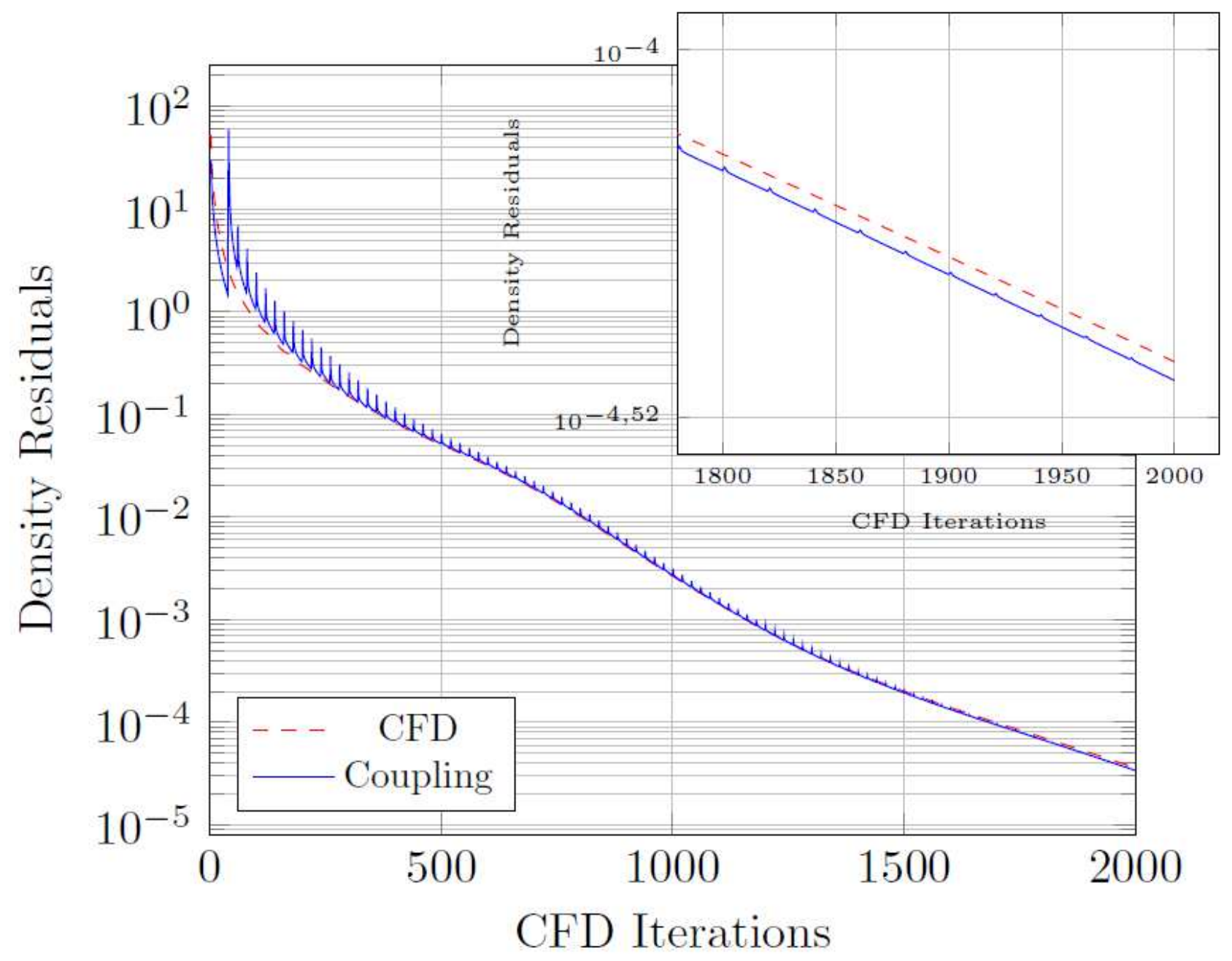

Figure 20. Comparison of convergence history between CHT and CFD only

The small peaks that can be observed are simply attributable to the updating of the boundary conditions during the CHT process $\left(\Delta t_{c}=20 \Delta t_{f}\right)$. Apart from these small differences, the two curves indicate the same tendencies. One even notices that CHT process based on the optimal coefficient is a bit quicker than the CFD only, as shown by the zoomed view in Figure 20. Here we compare only the number of CFD iterations necessary to converge without taking into consideration the solid iterations and the time taken for the MPI exchanges. This result can be explained by the fact that a temperature imposed in the CFD computation represents a perfect conducting condition while in the CHT computation, an ideal and local thermal conductance has been implemented at the F-S interface. 


\section{Strong Thermal Fluid-Structure Interaction}

\subsection{Amplification factors}

The preceding results clearly show that, for a given transmission condition scheme (in this case Dirichlet-Robin), it is possible to optimize the numerical procedure in terms of stability and convergence. This optimization is already a significant achievement. However, as stated before, it is useful to know whether the Dirichlet-Robin procedure is the most appropriate scheme to be used. In the following test case, we will show that this procedure and the resulting optimal coefficient (Eq. (10)) are not always appropriate.

Stability issues with the Dirichlet-Robin interface condition arise when the mesh Biot number $B i^{(\Delta)}$ is too large. This is illustrated in Figure 21 where a zoomed view of the amplification factor is represented for four different thermal conductivities of increasingly insulating materials, i.e. reducing $\lambda_{s}$, chosen for analysis.
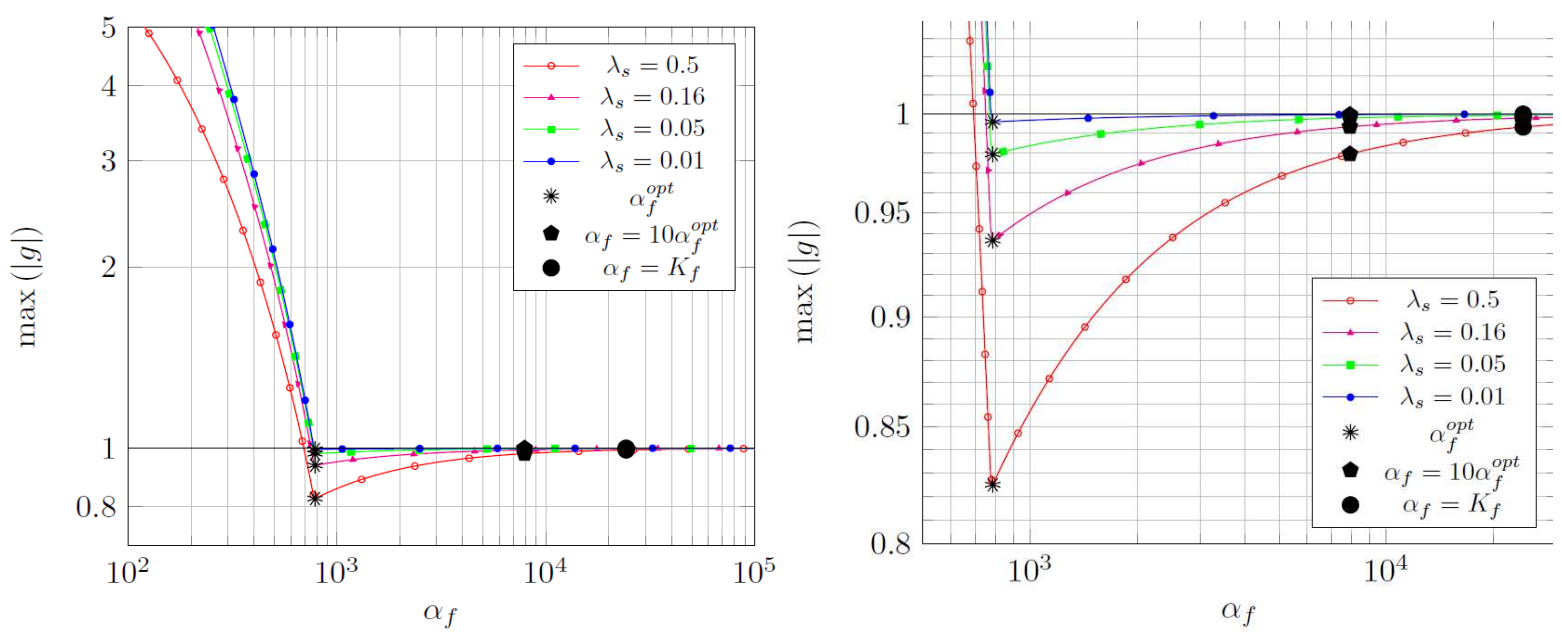

Figure 21. Amplification factor for three strong thermal interaction cases

As expected, the amplification factor in weak thermal interaction cases (Figure 10) is appreciably lower (or slightly higher in the first part of the curve) than unity. However, it is the opposite for a strong thermal interaction where the mesh Biot number has a very high value. In this case, the minimum of the amplification factor (the optimal coefficient) is close to unity. As a result, the CFD model induces a little destabilizing effect shifting it into the unstable zone. The minimal value of $\max \{|g|\}$ can be calculated by:

$$
\max _{|z|=1}\{|g|\}==\frac{\frac{B i^{(\Delta)}}{2}\left(1-\bar{D}_{f}\right)}{1+\frac{B i^{(\Delta)}}{2}\left(1-\bar{D}_{f}\right)}
$$

This high value indicates that a Dirichlet condition may be inappropriate. Of course, it can always be stabilized, as shown theoretically (through the time-dependent term $\left(1-\bar{D}_{f}\right)$ ). However, this stabilization might come at a high cost and sometimes it is not possible because of destabilizing 
effects induced by the flow, as mentioned previously. In this case, a natural physics-based approach consists in imposing a Neumann condition on the fluid side for strong thermal interaction.

\subsection{Comparison between the optimal Dirichlet-Robin and the Neumann-Dirichlet interface condition}

In Table 5 the numerical properties for Dirichlet-Robin and Neumann-Dirichlet interface conditions and the convergence iterations needed to converge, are shown.

\begin{tabular}{|c|c|c|c|c|c|c|}
\hline \multicolumn{3}{|c|}{ Parameters } & \multicolumn{3}{c|}{ Iterations to converge $\left(\varepsilon=10^{-3}\right)$} \\
\cline { 5 - 7 }$\lambda_{s}$ & $K_{s}$ & $\begin{array}{c}B i^{(\Delta)} \\
\text { Eq.(13) }\end{array}$ & $\begin{array}{c}B i_{v} \\
\text { Eq.(14) }\end{array}$ & $\alpha_{f}=\alpha_{f}^{(\text {opt })}$ & $\alpha_{f}=K_{f}$ & $\alpha_{f}=\infty$ \\
\hline 0.5 & 166. & 141. & 9.4 & 84 & 591 & 103 \\
\hline 0.16 & 53. & 446. & 28.9 & 273 & 815 & 105 \\
\hline 0.05 & 16.6 & 1645. & 86.8 & oscillations & 830 & 109 \\
\hline 0.01 & 3.3 & 7161. & 428. & $\begin{array}{c}\text { Crash at the } 13^{\text {th }} \\
\text { iteration }\end{array}$ & 516 & 104 \\
\hline
\end{tabular}

Table 5 : Numerical proprieties and convergence iteration at $10^{-3}$ in the cases with strong thermal interaction for the two boundary conditions. $\alpha_{s}$ is the coupling coefficient on the fluid side.

Figures 22, 23, 24 and 25 show the residual of the Neumann-Dirichlet (N-D) procedure as well as the convergence history of the optimal Dirichlet-Robin condition. 


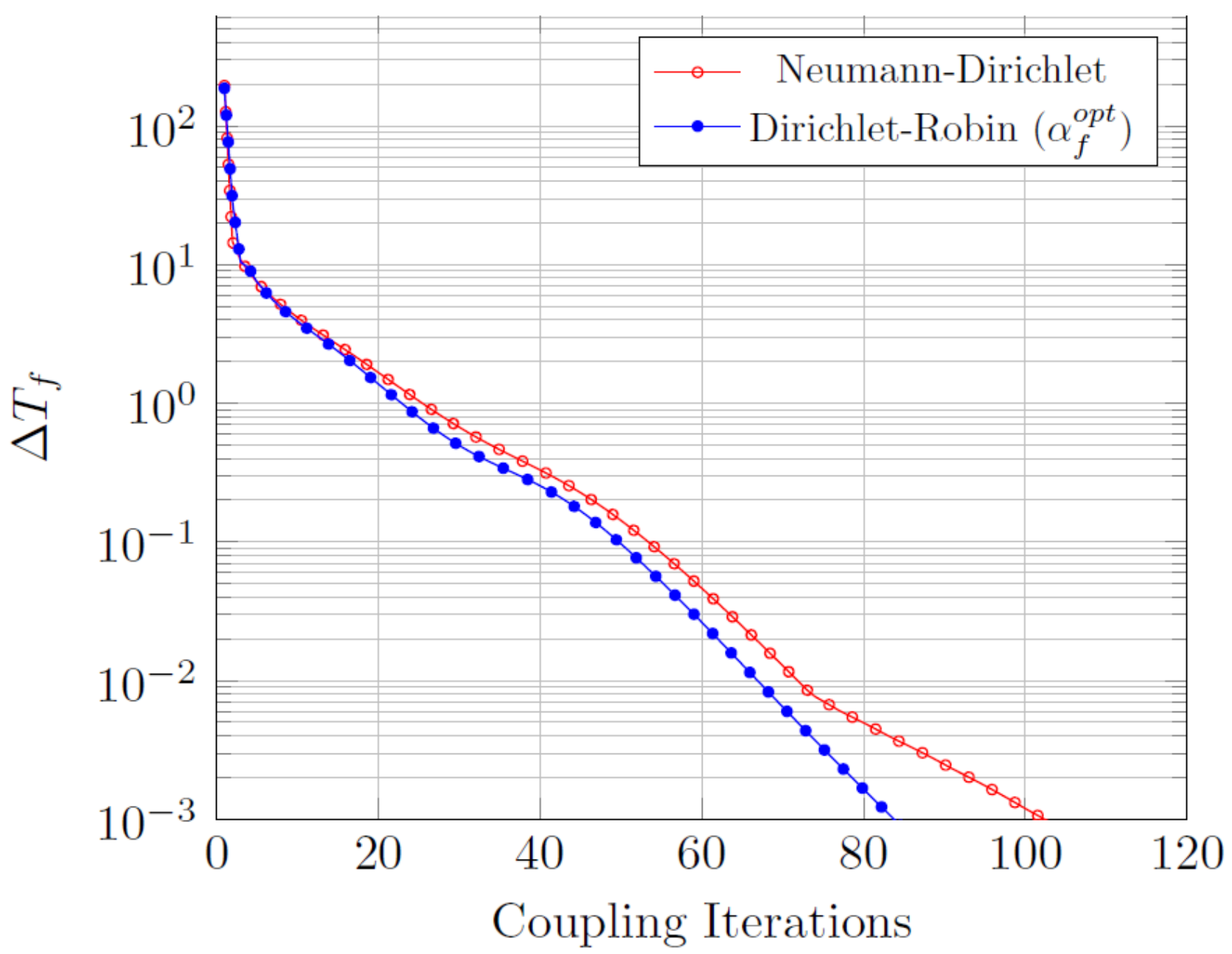

Figure 22. Convergence $v s$ coupling iterations $\left(\lambda_{s}=0.5 \mathrm{~W} \cdot \mathrm{m}^{-1} \cdot \mathrm{K}^{-1}-B i_{v}=9.4\right)$ 


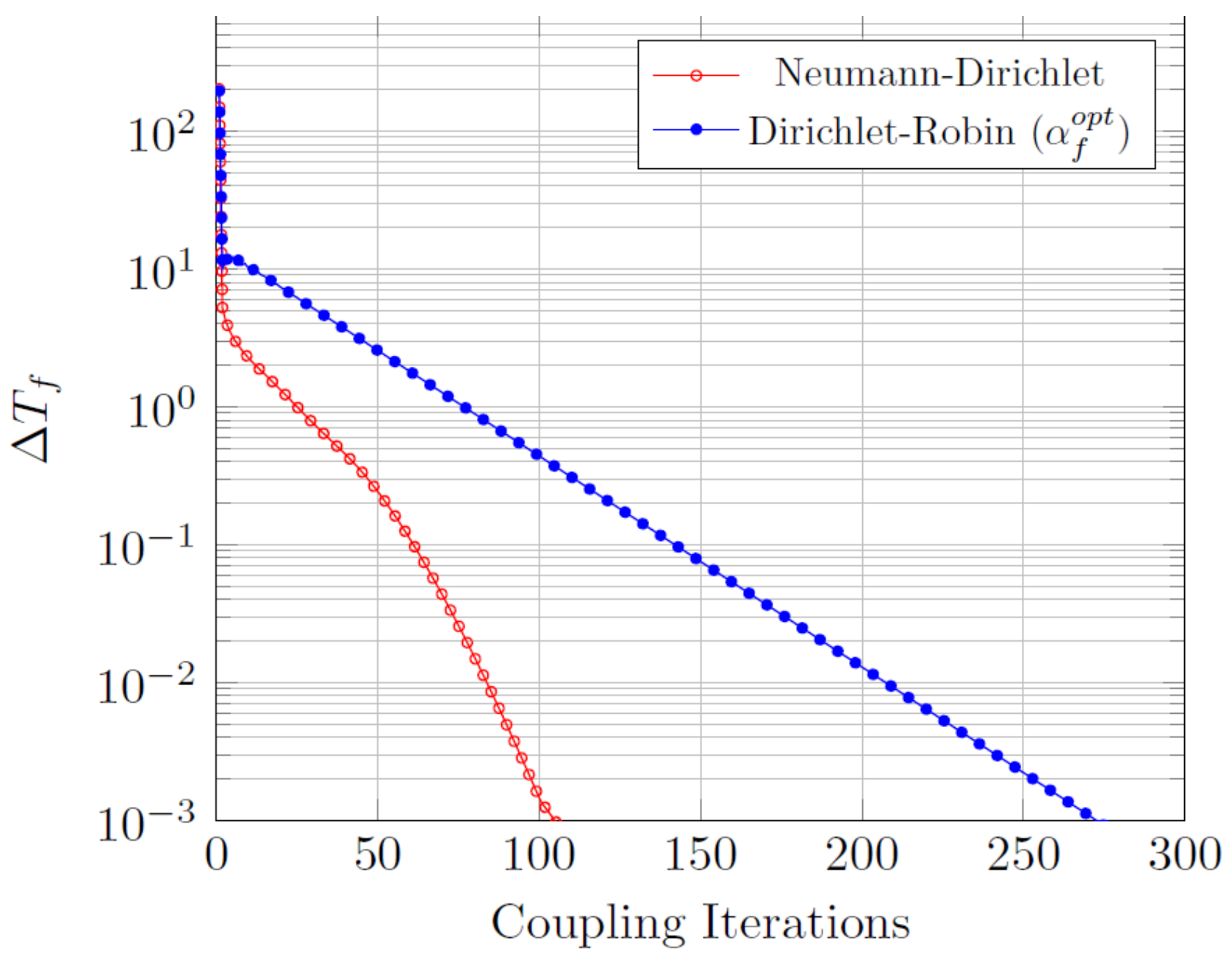

Figure 23. Convergence $v s$ coupling iterations $\left(\lambda_{\mathrm{s}}=0.16 \mathrm{~W} \cdot \mathrm{m}^{-1} \cdot \mathrm{K}^{-1}-B i_{v}=28.9\right)$ 


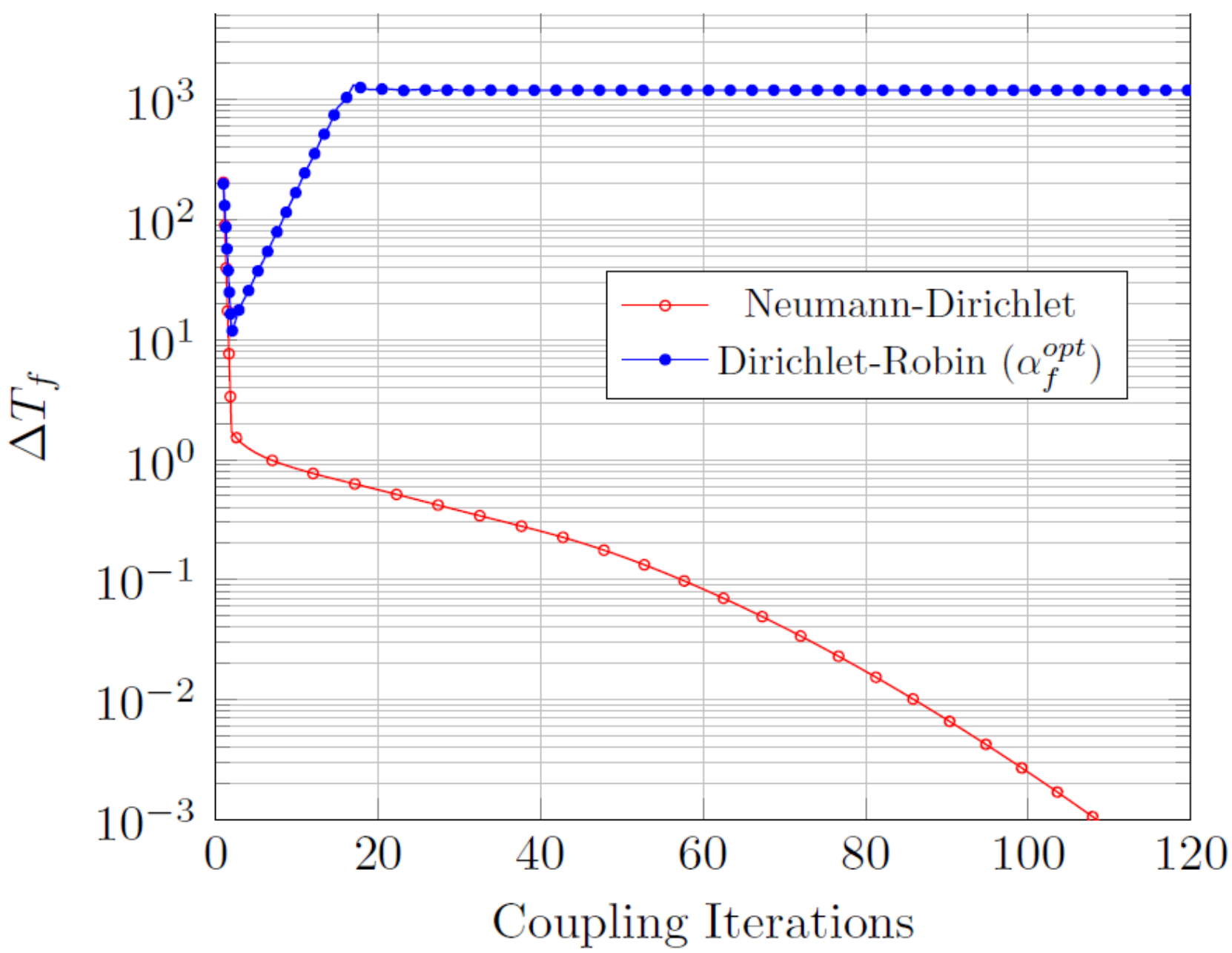

Figure 24. Convergence $v s$ coupling iterations $\left(\lambda_{\mathrm{s}}=0.05 \mathrm{~W} \cdot \mathrm{m}^{-1} \cdot \mathrm{K}^{-1}-B i_{v}=86.8\right)$ 


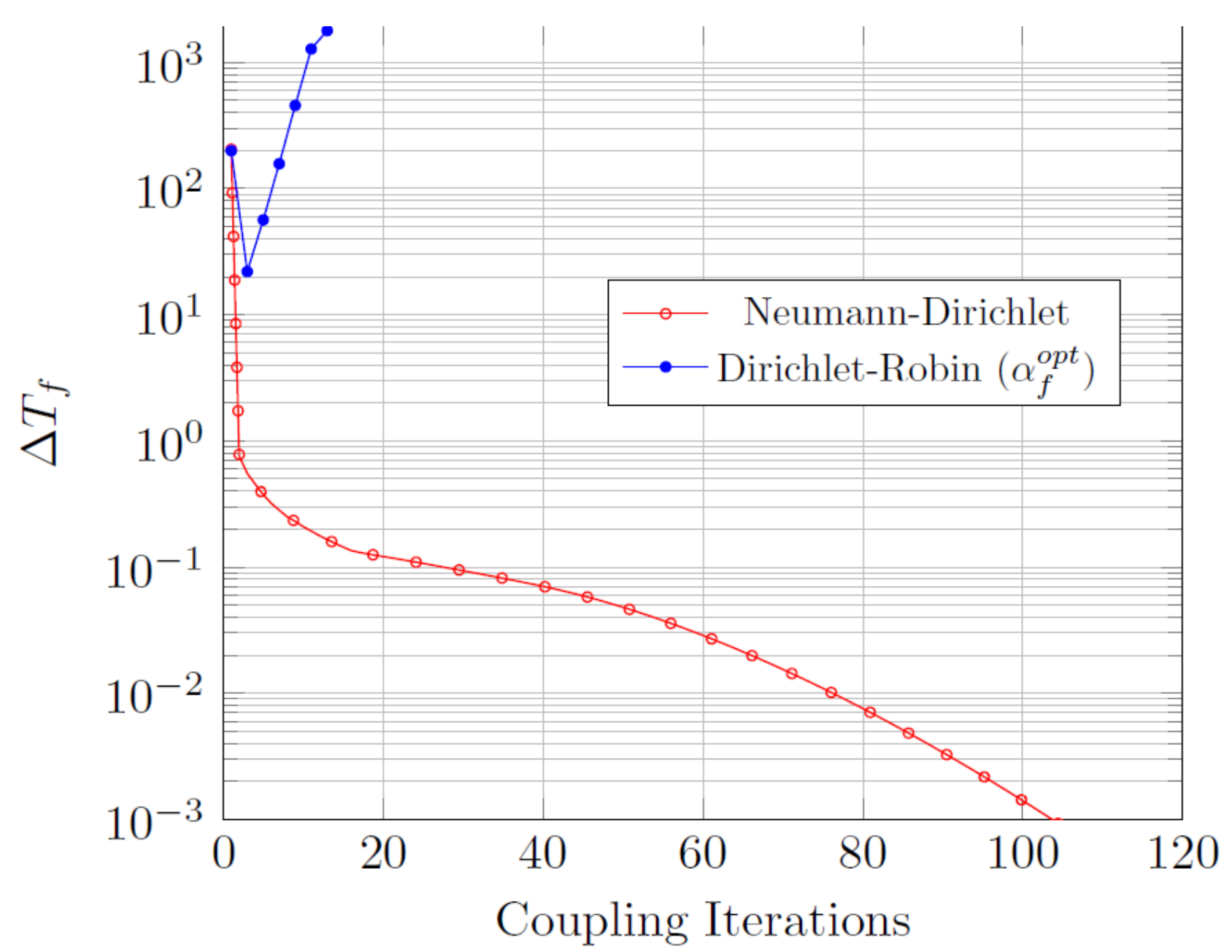

Figure 25. Convergence $v s$ coupling iterations $\left(\lambda_{\mathrm{s}}=0.01 \mathrm{~W} \cdot \mathrm{m}^{-1} \cdot \mathrm{K}^{-1}-B i_{v}=428.\right)$

Figure 22 shows that for a moderate thermal interaction, the choice of the optimal DirichletRobin is still the most efficient. However, if the mesh Biot number rises again, a degradation of the efficiency of the optimal Dirichlet-Robin interface condition is observed compared to the Neumann -Dirichlet one (Figure 23). This drop in performance becomes significant (273 coupling iterations are necessary for convergence for the optimal Dirichlet-Robin interface condition instead of 105 for the Neumann-Dirichlet condition). As the mesh Biot number increases further, the optimal Dirichlet-Robin does not achieve convergence (the temperature oscillates with an amplitude of $1000 \mathrm{~K}$ at every coupling iteration) while the N-D condition is rapidly convergent (see Figure 24). For very high mesh Biot numbers, the performance of the N-D conditions remains almost the same, whereas the optimal Dirichlet-Robin condition diverges as shown in Figure 25.

As a result, a Neumann-Dirichlet interface condition is more suitable to stabilize strong thermal interaction problems. These CHT computations highlight the importance of a relevant transmission procedure taking into account the heterogeneous nature of complex systems. Let us recall that the Neumann-Dirichlet procedure works without relaxation since there is no optimal coefficient for this condition at large numerical Biot number, as indicated by Table 2 and it shows the fastest convergence properties, when used appropriately for extremely large mesh Biot numbers. 


\section{CONCLUSION}

A simplified model problem was used as a basis to present the general expression of the amplification factor, the stability bounds, the optimal coefficient and the general numerical characteristics for two different interface procedures. It was shown that the numerical properties depend on the ratio of the fluid and solid thermal resistances and that the mesh Fourier number plays a crucial role. Furthermore, a numerical Biot number was introduced, and this number, in conjunction with the optimal coefficient, control the overall coupling process.

The conditions that establish the nature and character of the most relevant interface approach were identified and expressed. The numerical Biot number can clearly be regarded as a good criterion to choose the most appropriate physics-based approach. This number characterizes the strength of the thermal fluid-structure interaction and guides the CHT process as long as transient effects prevail. This number is based on the thermal response of the first point of the boundary layer which directly participates in the coupling process. Consequently, the resulting coupling coefficients are reliable from the very initial instants in the fluid domain until convergence.

Two possible scenarios were used to demonstrate the disparate thermal fluid-structure interaction provided by the $\mathrm{CHT}$ test case. As long as the numerical Biot number remains low or moderate, it was shown that the so-called optimal coefficient provides the best results in terms of stability and convergence in the Dirichlet-Robin procedure. This strategy can thus be used, regardless of the temporal evolution of the fluid domain, even when strong transient fluid effects are predominant. This adaptive and local coefficient makes it possible to avoid arbitrary relaxation coupling parameters. This strategy always gives stable and oscillation-free coupled solutions for this kind of fluid-structure interaction. Moreover, it is remarkable to note that the CHT computation time is even shorter than the time required for a CFD only computation in terms of fluid iterations.

For higher values of the numerical Biot number, or in other words when thermal gradients within the solid become important, a Dirichlet condition on the fluid side can no longer be considered as a physics-based approach. In this case, a Neumann condition is a suitable interface condition presenting good numerical properties with no need for relaxation coefficients. This was shown theoretically in this paper and confirmed by the test cases. As a result, a strategy based on these two complementary conditions was presented. Great numerical efficiency and substantial savings in computing time could be attained by using an appropriate interface transmission condition instead of being limited to a single procedure.

\section{Acknowledgment}

We would like to acknowledge the Direction Scientifique Générale de l'ONERA for their support. We also extend sincere and warm thanks to Andrew Mayne for his thorough re-reading of this article. 


\section{REFERENCES}

[1] T.L. Perelman, On conjugated problems of heat transfer, Int. J. Heat Mass Transfer 3 (1961) 293-303. https://doi.org/10.1016/0017-9310(61)90044-8

[2] A.V. Luikov, T.L. Perelman, R.S. Levitin, L.B. Gdalevich, Heat transfer from a plate in a compressible gas flow, Int. J. Heat Mass Transfer 13 (1971) 1261-1270. https://doi.org/10.1016/0017-9310(70)900670

[3] A. Matalip, N. Wansophark, P. Dechaumphai, Combined streamline Upwind Petrov Galerkin Method and segregated finite element algorithm for conjugate heat transfer problems, J. Mech. Sci. Technol. 20 (2006) 1741-1752. https://doi.org/10.1016/0017-9310(61)90044-8

[4] A. Matalip, N. Wansophark, P. Dechaumphai, A second-order time accurate finite element method for analysis of conjugate heat transfer between solid and unsteady viscous flow, J. Mech. Sci. Technol. 23 (2009) 775-789. doi:10.1007/s12206-008-1115-0

[5] C.A. Felippa, K.C. Park, Staggered transient analysis procedures for coupled dynamic systems: formulation, Comput. Methods Appl. Mech. Eng. 24 (1980) 61-111. https://doi.org/10.1016/0045-7825(80)90040-7

[6] P. Le Tallec, Domain decomposition methods in computational mechanics, Comput. Mech. Adv. 1 (1994) 121-220.

[7] S. Piperno, C. Farhat, B. Larrouturou, Partitioned procedures for the transient solution of coupled aroelastic problems Part I: Model problem, theory and two-dimensional application, Comput. Methods Appl. Mech. Eng. 124 (1995) 79-112. https://doi.org/10.1016/0045-7825(95)92707-9

[8] R. Jaiman, P. Geubelle, E. Loth, Stable and accurate loosely-coupled scheme for unsteady fluidstructure interaction, in: AIAA Aerospace Sciences Meeting and Exhibit, AIAA 2007-334, 2007. https://doi.org/10.2514/6.2007-334

[9] R. Jaiman, P. Geubelle, E. Loth, X. Jiao, Combined interface boundary conditions method for unsteady fluid-structure interaction, Comput. Methods Appl. Mech. Eng. 200 (2011) 27-39. https://doi.org/10.1016/j.cma.2010.06.039

[10] A. Yenduri, R. Ghoshal, R. Jaiman, A new partitioned staggered scheme for flexible multibody interactions with strong inertial effects, Comput. Methods Appl. Mech. Eng. 315 (2017) 316-347. doi:10.1016/j.cma.2016.10.044

[11] J. W. Banks, W. D. Henshaw, D. W. Schwendeman, An analysis of a new stable partitioned algorithm for FSI problems. Part I: Incompressible flow and elastic solids, J. Comput. Phys. 269 (2014) 108-137. doi:10.1016/j.jcp.2014.03.006

[12]S. Patankar, Numerical Heat Transfer and Fluid Flow, Mc Graw-Hill, 1980. doi:10.1002/cite. 330530323

[13] M. Schäfer, I. Teschauer, Numerical simulation of coupled fluid-solid problems, Comput. Methods Appl. Mech. Eng. 190 (2001) 3645-3667. https://doi.org/10.1016/S0045-7825(00)00290-5

[14]X. Chen, P. Han, A note on the solution of conjugate heat transfer problems using SIMPLE-like algorithms, Int. J. Heat Fluid Flow 21 (2000) 463-467. doi:10.1016/S0142-727X(00)00028-X

[15] G. Comini, O. Saro, M. Manzan, A physical approach to finite element modeling of coupled conduction and convection, Numer. Heat Transfer, Part B 24 (1993) 243-261. doi: 10.1080/10407799308955892 
[16]W. Shyy, J. Burke, Study of iterative characteristics of convective diffusive and conjugate heat transfer problems, Numer. Heat Transfer, Part B 21-37. http://dx.doi.org/10.1080/10407799408914914

[17] K.H. Kao, M.S. Liou, Application of Chimera/unstructured hybrid grids for conjugate heat transfer, AIAA J. 35 (1997) 1472-1478. https://doi.org/10.2514/2.270

[18] P. Causin, J.F. Gerbeau, F. Nobile, Added-mass effect in the design of partitioned algorithms for fluidstructure problems, Comput. Methods Appl. Mech. Eng. 194 (2005) 4506-4527. doi:10.1016/j.cma.2004.12.005

[19] J.W. Banks, B. Sjogreen, A normal mode stability analysis of numerical interface conditions for fluid/structure interaction, Comm. Comput. Phys. 10 (2011) 279-304. doi:10.4208/cicp.060210.300910a

[20] M.B. Giles, Stability analysis of numerical interface conditions in fluid-structure thermal analysis, Int. J. Numer. Methods Fluids 25 (1997) 421-436. doi:10.1002/(SICI)1097-0363(19970830)25:4<421::AIDFLD557>3.0.CO;2-J

[21]B. Roe, R. Jaiman, A. Haselbacher, P.H. Geubelle, Combined interface boundary method for coupled thermal simulations, Int. J. Numer. Methods Fluids 57 (2008) 329-354. http://doi.wiley.com/10.1002/fld.1637

[22] W.D. Henshaw, K.K. Chand, A composite grid solver for conjugate heat transfer in fluid-structure systems, J. Comput. Phys. 228 (2009) 3708-3741. https://doi.org/10.1016/j.jcp.2009.02.007

[23] V. Kazemi-Kamyab, A.H. van Zuijlen, H. Bijl, Accuracy and stability analysis of a second-order timeaccurate loosely coupled partitioned algorithm for transient conjugate heat transfer problems, Int. J. Numer. Methods Fluids 74 (2014) 113-133. doi:10.1002/fld.3842

[24]T. Verstraete, S. Scholl, Stability analysis of partitioned methods for predicting conjugate heat transfer, Int. J. Heat Mass Transfer 101 (2016) 852-869. doi:10.1016/j.ijheatmasstransfer.2016.05.041

[25] J. Lindström, J. Nordström, A stable and high-order conjugate heat transfer problem, J. Comput. Phys. 229 (2010) 5440-5456. doi:10.1016/j.jcp.2010.04.010

[26] M.P. Errera, S. Chemin, Optimal solutions of numerical interface conditions in fluid-structure thermal analysis, J. Comput. Phys. 245 (2013) 431-455. doi:10.1016/j.jcp.2013.03.004

[27] S.K. Godunov, V.S. Ryabenkii, The theory of difference schemes: an introduction, North-Holland, Amsterdam, 1964. doi: 10.1126/science.148.3671.811-a

[28] H.O. Kreiss, Stability theory for difference approximations of mixed initial boundary value problems. 1, Math. Comput. 22 (1968) 703-714. doi:10.2307/2004572

[29]B. Gustafsson, The Godunov-Ryabenkii condition: the beginning of a new stability theory, Technical report / Department of Information Technology, Uppsala University, 1999. https://doi.org/10.1007/978$1-4615-0663-8 \_42$

[30] M.P. Errera, G. Turpin, Temporal multiscale strategies for conjugate heat transfer problems, J. Coupled Syst. Multiscale Dyn. 1 (2013) 89-98. doi:10.1166/jcsmd.2013.1005

[31] M.P. Errera, F. Duchaine, Comparative study of coupling coefficients in Dirichlet-Robin procedure for fluid-structure aerothermal simulations, J. Comput. Phys. 312 (2016) 218-234. doi:10.1016/j.jcp.2016.02.022

[32]R. El Khoury, M.P. Errera, K. El Khoury, M. Nemer, Efficiency of coupling schemes for the treatment of steady state fluid-structure thermal interactions, Int. J. Therm. Sci. 115 (2017) 225-235. doi:10.1016/j.ijthermalsci.2017.02.001 
[33] M.P. Errera, M. Lazareff, J.D. Garaud, T. Soubrié, C. Douta, T. Federici, A coupling approach to modeling heat transfer during a full transient flight cycle, Int. J. Heat Mass Transfer 110 (2017) 587605. doi:10.1016/j.ijheatmasstransfer.2017.03.048

[34] S. Badia, F. Nobile, C. Vergara, Fluid-structure partitioned procedures based on Robin transmission conditions, J. Comput. Phys. 227 (2008) 7027-7051. doi:10.1016/j.jcp.2008.04.006

[35] F. Meng, J.W. Banks, W.D. Henshaw, D.W. Schwendeman, A stable and accurate partitioned algorithm for conjugate heat transfer, J. Comput. Phys. 344 (2017) 51-85. doi:10.1016/j.jcp.2017.04.052

[36] A. Montenay, L. Paté, J.M. Duboué, Conjugate heat transfer analysis of an engine internal cavity, in: ASME Turbo Expo, ASME 2000-GT-282, 2000. http://dx.doi.org/10.1115/2000-GT-0282

[37]E. Divo, E. Steinthorsson, A.J. Kassab, R. Bialecki, An iterative BEM/FVM protocol for steady-state multi-dimensional conjugate heat transfers in compressible flows, Eng. Anal. Boundary Elem. 26 (2002) 447-454. https://doi.org/10.1016/S0955-7997(01)00106-0

[38] A.R. Crowell, B.A. Miller, J.J. McNamara, Computational modeling for conjugate heat transfer of shock-surface interactions on compliant skin panels, in: AIAA/ASME/ASCE/AHS/ASC Structures, Structural Dynamics and Materials Conference, AIAA 2011-2017, 2011. https://doi.org/10.2514/6.2011-2017

[39] J. Heidman, D. Rigby, A. Ameri, A three-dimensional coupled external-internal simulation of a film cooled turbine vane, ASME J. Turbomach 124 (2000) 348-359. doi:10.1115/1.555450

[40] A. Heselhaus, D.T. Vogel, Numerical simulation of turbine blade cooling with respect to blade heat conduction and inlet temperature profiles, in: AIAA/ASME/ASEE Joint Propulsion Conference and Exhibit, AIAA 95-3041, 1995. https://doi.org/10.2514/6.1995-3041

[41] R.S. Amano, K.D. Wang, V. Pavelic, A study of rotor cavities and heat transfer in a cooling process in a gas turbine, ASME J. Turbomach. 116 (1994) 333-338. http://dx.doi.org/10.1115/1.2928369

[42]J.A. Verdicchio, J.W. Chew, N.J. Hills, Coupled heat solid transfer computation for turbine disks, ASME Turbo Expo, ASME 2001-GT-0205, 2001. http://dx.doi.org/10.1115/2001-GT-0205

[43]Zset : Non-linear material \& structure analysis suite.

http://www.zset-software.com (accessed 25 October 2017)

[44] elsA : A CFD software package for compressible flows around complex various geometries. http://elsa.onera.fr (accessed 25 October 2017)

[45]L. Cambier, M. Gazaix, elsA : an efficient object-oriented solution to CFD complexity, in: AIAA Aerospace Science Meeting and Exhibit, AIAA 2002-0108, 2002. https://doi.org/10.2514/6.2002-108

[46]L. Cambier, S. Heib, S. Plot, The Onera elsA CFD software: input from research and feedback from industry, Mech. \& Ind. 14 (2013) 159-174. doi:10.1051/meca/2013056

[47]CWIPI : Coupling with Interpolation Parallel Interface. http://sites.onera.fr/cwipi (accessed 25 October 2017) 